\title{
Zeb2 recruits HDAC-NuRD to inhibit Notch and controls Schwann cell differentiation and remyelination
}

\author{
Lai Man Natalie Wu ${ }^{1}$, Jincheng Wang ${ }^{1,2}$, Andrea Conidi ${ }^{3}$, Chuntao Zhao ${ }^{1}$, Haibo Wang ${ }^{1}$, Zachary Ford ${ }^{4}$, \\ Liguo Zhang ${ }^{1}$, Christiane Zweier ${ }^{5}$, Brian G Ayee ${ }^{6}$, Patrice Maurel ${ }^{6}$, An Zwijsen ${ }^{7}$, Jonah R Chan ${ }^{8}$, \\ Michael P Jankowski ${ }^{4}$, Danny Huylebroeck ${ }^{3,9}$ \& Q Richard Lu ${ }^{1,10}$
}

The mechanisms that coordinate and balance a complex network of opposing regulators to control Schwann cell (SC) differentiation remain elusive. Here we demonstrate that zinc-finger E-box-binding homeobox 2 (Zeb2, also called Sip1) transcription factor is a critical intrinsic timer that controls the onset of SC differentiation by recruiting histone deacetylases HDAC 1 and 2 (HDAC1/2) and nucleosome remodeling and deacetylase complex (NuRD) co-repressor complexes in mice. Zeb2 deletion arrests SCS at an undifferentiated state during peripheral nerve development and inhibits remyelination after injury. Zeb2 antagonizes inhibitory effectors including Notch and Sox2. Importantly, genome-wide transcriptome analysis reveals a Zeb2 target gene encoding the Notch effector Hey2 as a potent inhibitor for Schwann cell differentiation. Strikingly, a genetic Zeb2 variant associated with Mowat-Wilson syndrome disrupts the interaction with HDAC1/2-NuRD and abolishes Zeb2 activity for SC differentiation. Therefore, Zeb2 controls SC maturation by recruiting HDAC1/2-NuRD complexes and inhibiting a Notch-Hey2 signaling axis, pointing to the critical role of HDAC1/2-NuRD activity in peripheral neuropathies caused by ZEB2 mutations.

\begin{abstract}
Schwann cells of the peripheral nervous system (PNS) ensheath and wrap myelin membranes around axons to maximize the efficiency of rapid saltatory conduction of action potentials. Functional defects of SCs contribute to various forms of peripheral neuropathies ${ }^{1-3}$. A recently defined autosomal dominant disorder, Mowat-Wilson syndrome (MOWS), is caused by mutations in ZEB2 (also called $S I P 1$ or $Z F H X 1 B)$, the gene that encodes zinc finger E-box-binding homeobox 2 protein. MOWS is characterized by a wide spectrum of congenital anomalies encompassing growth defects, intellectual disability, delayed motor development and neurocristopathies with defects in neural crest derivatives ${ }^{4-6}$. In mice, Zeb2 is critical for neurogenesis and gliogenesis in the $\mathrm{CNS}^{7-9}$. Recent studies indicate that Zeb2 can interact with NuRD, suggesting that NuRD association may be important to Zeb2 functions ${ }^{4,10}$. At present, whether and how Zeb2 controls SC differentiation and myelination remain elusive. Elucidation of Zeb2 functions in peripheral nerve cell development in addition to CNS neurogenesis may provide a better understanding of the etiology of neurological diseases associated with MOWS caused by ZEB2 mutations.
\end{abstract}

Development and myelination of neural-crest-derived SCs are coordinately regulated by a spectrum of extracellular signals and intrinsic regulators. These comprise positive cues including neuregulin 1 (NRG1)11,12 and laminin-integrin signaling ${ }^{13,14}$,
G-protein-coupled receptors (GPCRs) such as Gpr126 (ref. 15) and negative cues including Notch signaling and Sox2 (ref. 16). Timely initiation of SC myelination not only demands a strict interplay between extrinsic cues and intracellular inputs, but also requires the precise balance of the activity of an intricate network of transcriptional regulators. Transcription factors such as the high mobility group (HMG)-type protein Sox10, the Pit-Oct-Unc (POU)homeodomain proteins Oct6 (also called Pou3f1) and Pou3f2 (also called Brn2), and the zinc-finger proteins Krox20 (also called Egr2) and YY1 are crucial for promoting SC myelination ${ }^{17-20}$. How these multiple signaling pathways converge and coordinate the myelination program and, more importantly, what orchestrates the fine-tuning of proper transcriptional outputs are not fully understood.

Here, using SC-lineage-specific in vivo mutagenesis, we identified Zeb2 as a critical intrinsic timer controlling the onset of SC differentiation during normal myelination and also in remyelination after peripheral nerve injury. The predominant effects of Zeb2 during SC development are to directly repress a network of inhibitory pathways including Sox 2 and Notch-Hey2 signaling. We further find that a single amino acid variant in ZEB2 seen in a patient with MOWS disrupts interaction with the HDAC1/2-NuRD co-repressor complex and abolishes the ability of ZEB2 to effect SC differentiation. Our data thus establish a critical role for HDAC1/2-NuRD-dependent

\footnotetext{
${ }^{1}$ Department of Pediatrics, Division of Experimental Hematology and Cancer Biology, Cincinnati Children's Hospital Medical Center, Cincinnati, Ohio, USA. ${ }^{2}$ Institute of Pharmacology and Toxicology, College of Pharmaceutical Sciences, Zhejiang University, Hangzhou, China. ${ }^{3}$ Department of Cell Biology, Erasmus University Medical Center, Rotterdam, the Netherlands. ${ }^{4}$ Department of Anesthesiology, Cincinnati Children's Hospital Medical Center, Cincinnati, Ohio, USA. ${ }^{5}$ Institute of Human Genetics, Friedrich-Alexander-Universität Erlangen-Nürnberg, Erlangen, Germany. ${ }^{6}$ Department of Biological Science, Rutgers University, Newark, New Jersey, USA. 7VIB Center for the Biology of Disease, KU Leuven Department of Human Genetics, Leuven, Belgium. ${ }^{8}$ Department of Neurology, Program in Biomedical and Neurosciences, University of California, San Francisco, San Francisco, California, USA. ${ }^{2}$ Laboratory of Molecular Biology (Celgen), KU Leuven Department of Development and Regeneration, Leuven, Belgium. 10Key Laboratory of Birth Defects, Children's Hospital of Fudan University, Shanghai, China. Correspondence should be addressed to Q.R.L. (richard.lu@cchmc.org).
}

Received 18 March; accepted 10 May; published online 13 June 2016; corrected online 20 June 2016 (details online; doi:10.1038/nn.4322 
Zeb2 activity in initiating SC differentiation and myelination through opposing Notch- and Sox2-mediated negative regulatory circuitry.

\section{RESULTS}

\section{Dynamic Zeb2 expression during SC lineage development}

To examine Zeb2 expression in SCs, we coimmunolabeled for Zeb2 and for SC lineage marker Sox10 in developing sciatic nerves (Fig. 1a). The vast majority of Zeb2-positive cells were also Sox10-positive at postnatal day (P) 7. In keeping with its function as transcription regulator, Zeb2 was detected in the nuclei of Sox $10^{+}$SCs. Concurrent with the onset of myelination in the developing nerves, Zeb2 was highly expressed in SCs during the first 2 postnatal weeks, and the signal gradually declined as the animal reaches adulthood at 10 weeks (Fig. 1a).

To further define Zeb2 levels during SC lineage progression, we examined Zeb2 mRNA expression using SC-enriched sciatic nerves at different stages by real-time quantitative PCR (qPCR). Consistently, Zeb2 transcripts were detected at the neonatal stage $\mathrm{P} 0$ and peaked at the perinatal stage P10, but then were reduced in adulthood (Fig. 1b).

\section{Zeb2 mutant mice display severe motor deficits}

To determine the cell-autonomous function of Zeb2 in SC development, we generated conditional mutant mice lacking functional Zeb2 in SCs by breeding mice carrying a Zeb2 loxP-flanked allele $\left(Z e b 2^{\operatorname{lox} P / l o x P}\right)^{8,21}$ with a SC-expressing Cre line driven by the Desert hedgehog (Dhh) promoter ${ }^{17}$ (Fig. 1c). We used Zeb2 ${ }^{\text {loxP/+}} ;$ Dhh-cre or Zeb2 $2^{\text {loxP/loxP }}$ littermates as controls since they were phenotypically normal in SC development (Supplementary Fig. 1a-g).

Cre-mediated inactivation of Zeb2 efficiently abrogated Zeb2 presence in Sox $10^{+}$SCs in peripheral nerves (Fig. 1d). Zeb2 $2^{\text {loxP } / l o x P}$; Dhh-cre (hereafter referred to as Zeb2 cKO) mice were born in numbers predicted by Mendelian ratios. During the first postnatal week, mutant mice were essentially indistinguishable from littermate controls. Starting in the second week, however, all Zeb2 cKO mice began to develop severe tremor, unsteady gait and hindlimb paralysis, and the majority died by 8 weeks (Supplementary Fig. 2a).

Upon tail suspension, mutant mice at P21 showed abnormal hindlimb clasping (Fig. 1e). Zeb2 cKO sciatic nerves at P7 exhibited a thin and translucent appearance, in stark contrast to the thick, opaque nerves of control mice, suggesting a severe deficit at the onset of myelinogenesis (Fig. 1f). Consistent with the overt clinical signs of hypomyelination in the Zeb2 cKO mutants, the motor unit function of mutant mice was severely impaired, as reflected by a marked reduction in conduction velocity (Fig. 1g) measured by electrophysiological recordings of compound muscle action potentials (CMAPs) during electrical stimulation of the sciatic nerve in vivo. The nerve conduction velocity was reduced from $27.7 \pm 2.7 \mathrm{~m} \mathrm{~s}^{-1}$ in littermate controls to $1.8 \pm 0.1 \mathrm{~m} \mathrm{~s}^{-1}$ in the mutants at the young adult stage (P42), which is close to the level of the slower, thinly myelinated A $\delta$ or unmyelinated $\mathrm{C}$ fiber conduction velocity in mice ${ }^{22}$ (Fig. 1h). Furthermore, the mean peak amplitudes and duration of CMAPs
Figure 1 SC-specific deletion of Zeb2 results in peripheral nerve hypomyelination. (a) Co-localization of Zeb2 (green) with Sox10 (red) in SC nuclei from 1 to 10 weeks (wk) by immunofluorescence labeling. Scale bar, $100 \mu \mathrm{m}$. The experiment was repeated 3 times on 3 control and mutant tissues. (b) qPCR showing Zeb2 transcript levels in mouse sciatic nerves at various developmental age. $n=3$ control tissues at each time point; mean \pm s.e.m.; ${ }^{* *} P<0.001$; one-way ANOVA with multiple comparisons test; $P_{(\mathrm{P} 10)}=$ $0.0003, P_{(\mathrm{P} 20)}=0.0009, P_{(\mathrm{P} 60)}<0.0001$; $F_{3,12}=61.91$. (c) Exon 7 of the loxP-flanked Zeb2 allele is excised by Dhh-cre. (d) Colabeling of Zeb2 (green) with Sox10+ (red) SC nuclei in mutant sciatic nerves at P7. Scale bar, $50 \mu \mathrm{m} ; n=3$ control and mutant tissues. (e) Tail suspension test showing abnormal hindlimb reflex in Zeb2 cKO (Zeb2loxP/loxP; Dhh-cre+/-) but not in a control littermate (Zeb2/oxP/+; Dhh-Cre ${ }^{+/-}$) at P21. (f) Sciatic nerves from control and Zeb2 cKO mice at P7. (g) CMAPs from P42 control and Zeb2 cKO. S, stimulus (black arrows); R, initiation of CMAP response (red arrows). (h) Nerve conduction velocities in Zeb2 cKO (mutant) and control littermates at P42. Data are presented as mean \pm s.e.m. $n=4$ control and mutant tissues; ${ }^{* *} P<0.001, t=9.538$, d.f. $=6$; two-tailed unpaired Student's $t$-test. (i) Mean peak amplitudes of CMAPs in sciatic nerves of control and Zeb2 cKO (mutant) mice at P42. Data are presented as mean \pm s.e.m. $n=4$ control and mutant tissues; ${ }^{*} P<0.05 ; P=0.0417$, $t=2.582$, d.f. $=6$; two-tailed unpaired

Student's $t$-test. (j) Average durations of CMAPs in sciatic nerves of control and Zeb2 cKO (mutant) mice at P42. Data are presented as mean \pm s.e.m. ( $n=4$ control and mutant tissues; ${ }^{*} P<0.05 ; P=0.0155, t=3.348$, d.f. $=6$; two-tailed unpaired Student's $t$-test). a
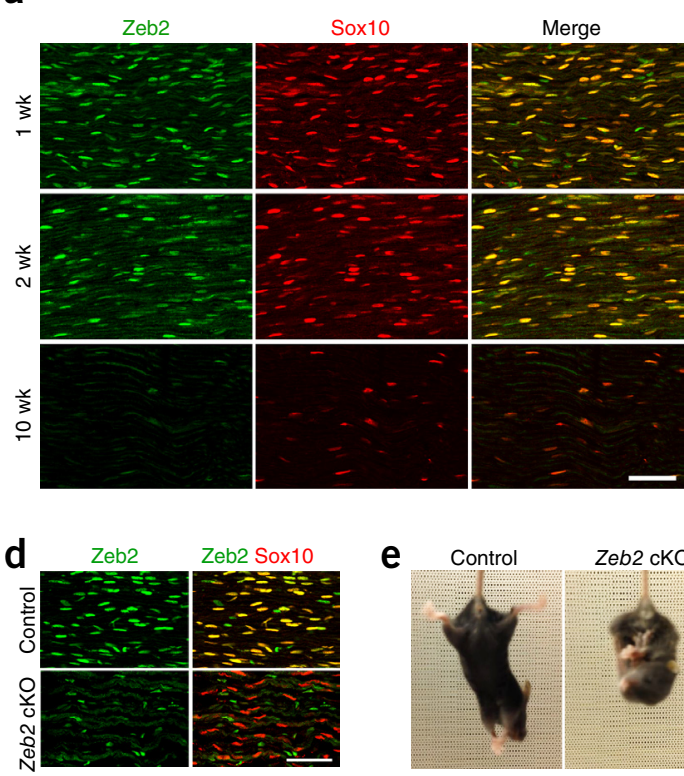

$\mathbf{e}$

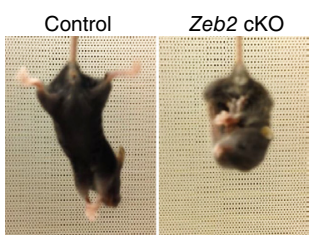

g

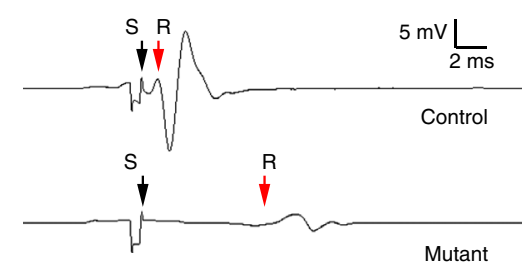

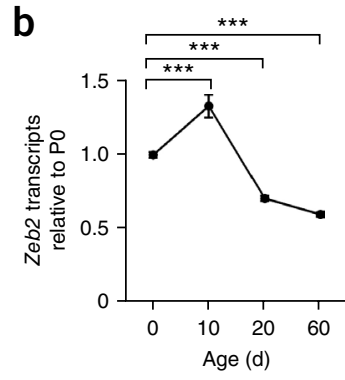

C Zeb2 loxP-flanked allele Exon $\begin{array}{llll}45 & 6 & 7 & 8\end{array}$ $\begin{array}{lll}\text { IXxP loxP } & \text { SC-expressing } \\ \text { Exon } 4568 & \text { Dhh-cre } \\ & \end{array}$ \#-14 f Control Zeb2 cKO HIt

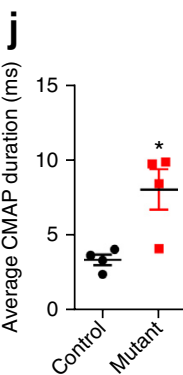


were also severely affected in Zeb2 cKO mice (Fig. 1i,j), accounting for the defect in motor function in Zeb2 mutants.

\section{Zeb2-deficient SCs fail to myelinate in peripheral nerves}

We evaluated myelinogenesis in Zeb2 cKO peripheral nerves by myelin basic protein (MBP) immunolabeling. In contrast to the robust expression in control nerves, $\mathrm{MBP}$ was scarcely expressed in the mutant sciatic nerves at P14 (Fig. 2a). Accordingly, expression of the major myelin genes $M b p$ and $M p z$ in sciatic nerves was virtually undetectable in mutants by in situ hybridization (Fig. 2b) and qPCR (Fig. 2c). Ultrastructural examination by electron microscopy (EM) revealed that, whereas by $\mathrm{P} 4$ control SCs had established a 1:1 relationship with large-caliber axons and begun wrapping myelin membrane around axons, the majority of mutant SCs were associated with multiple axons and failed to initiate myelination, indicative of incomplete axonal sorting and a dysmyelination phenotype (Fig. 2d). At P21, axon bundles ensheathed by Zeb2 cKO SCs remained essentially unsorted and unmyelinated (Fig. 2e,f), despite apparent normal SC process extension around associated axons (Fig. 2f). Moreover, small-diameter axons remained unsegregated in axon bundles with large diameter axons, further confirming sorting defects in Zeb2 cKO (Supplementary Fig. 1c). However, in testing for cutaneous thermal hypersensitivity, Zeb2 cKO showed a similar decrease in withdrawal latency to $50{ }^{\circ} \mathrm{C}$ water following tissue inflammation compared to their baseline responsiveness, as was seen with littermate controls (Supplementary Fig. 1h).
Very few mutant mice survived to adulthood (Supplementary Fig. 2a). The gross structure of peripheral nerves in rare Zeb2 $\mathrm{cKO}$ survivors at 2 months showed minifascicles of SCs and axons with no apparent sign of myelination, suggesting that Zeb2 loss in SCs leads to a sustained myelination block in peripheral nerves (Fig. 2g).

To bypass the possible influence of the Zeb2 loss during early SC development, we inactivated Zeb2 in the peripheral nerves of neonates using a tamoxifen-inducible $P l p 1-c r e^{E R T}$ driver $^{23}$ with tamoxifen administration from P0 to P4 (Fig. 2h). By P14, more than half of the large axons were not properly sorted and remained unmyelinated in Zeb2 inducible knockout (iKO; Zeb2 $\left.2^{\text {loxP/loxP }} ; P l p 1-c r e^{E R T}\right)$ mutant nerves (Fig. 2i,j). The ultrastructural observations seen upon Zeb2 deletion in immature SCs suggest that Zeb2 is required for proper initiation of SC differentiation and myelination.

The diminished expression of Zeb2 in mature nerves (Fig. 1a,b) suggested a nonobligatory function in myelin maintenance. Accordingly, tamoxifen-induced deletion of Zeb2 in adult mice altered neither the myelin sheath's thickness nor its integrity in peripheral nerves (Supplementary Fig. 2b), suggesting a crucial role for Zeb2 in initiating or priming SC differentiation rather than maintaining myelin sheaths.

\section{Zeb2 controls myelinogenic programs for SC differentiation}

In Zeb2 cKO sciatic nerves at P7, we detected reduced levels of mRNAs encoding SC prodifferentiation regulators including Sox10, Krox20 and Oct6 (Fig. 3a). In contrast, among known negative regulators, we
Figure 2 Zeb2 is required for SC myelination. (a) MBP staining in P14 control and Zeb2 cKO longitudinal sciatic nerves ( $n=3$ control and mutant tissues). Scale bars, $50 \mu \mathrm{m}$.

(b) Expression of $M b p$ and $M p z$ in transverse sections of sciatic nerves from control and Zeb2 cKO mice ( $n=3$ control and mutant tissues) at P21 by in situ hybridization. Scale bars, $200 \mu \mathrm{m}$. (c) qPCR showing the levels of myelinrelated genes in sciatic nerves of $\mathrm{P} 7$ control and Zeb2 cKO mice. Data are presented as mean \pm s.e.m. ${ }^{* * *} P<0.0001, n=3$ control and 3 mutant tissues, $P_{(P r x)}<0.0001, t=2,750$, d.f. $=4 ; P_{(M b p)}<0.0001, t=512.5$, d.f. $=4$; $P_{(M p z)}<0.0001, t=1,088$, d.f. $=4$; two-tailed unpaired Student's $t$-test. (d,e) Representative EM images showing ultrastructure of control and Zeb2 cKO sciatic nerves at P4 (d) and P21 (e). $n=3$ control and mutant tissues. Arrowheads indicate axons and SC-axon units, respectively. Scale bars, $2 \mu \mathrm{m}$. (f) EM images showing a 1:1 SC-axon unit in a P21 control sciatic nerve (top) and a SC (S) associating with multiple axons in a mutant nerve (bottom). $n=3$ control and mutant tissues. Arrow indicates mutant SC process surrounding an axon. Scale bars, $500 \mathrm{~nm}$. (g) Representative EM images of P60 control and Zeb2 cKO sciatic nerves. $n=3$ control and mutant tissues. Scale bars, $2 \mu \mathrm{m}$. (h) Tamoxifen (TAM) administration scheme: pups were injected with TAM via intraperitoneal injections (i.p.) into lactating dams once per day from $\mathrm{PO}$ to $\mathrm{P} 4$, and sciatic nerves were collected on P14. (i) EM images of cross sections of P14 sciatic nerves from control (Zeb2loxP/+ $;$ PIp1-cre ${ }^{E R T} ; n=3$ ) and Zeb2 iKO mice (Zeb2loxP/loxP; Plp1-cre $\left.{ }^{E R T} ; n=4\right)$ induced by TAM from PO-4. Arrows denote incompletely sorted axon bundles. Scale bars, $2 \mu \mathrm{m}$. (j) Myelinated axons per cross section of P14 sciatic nerves from control and TAM-treated Zeb2 iKO. Data are presented as mean \pm s.e.m.; $n=3$ controls and 4 mutants; two-tailed unpaired Student's $t$-test. ${ }^{* *} P<0.01 ; P=0.0062, t=4.529$; d.f. $=5$.
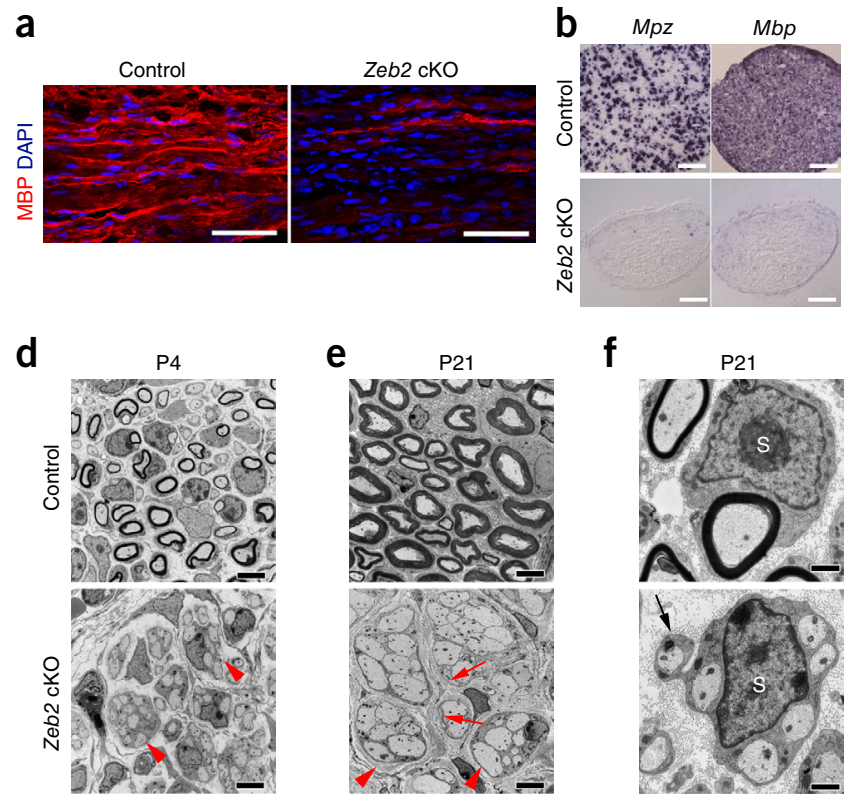

e

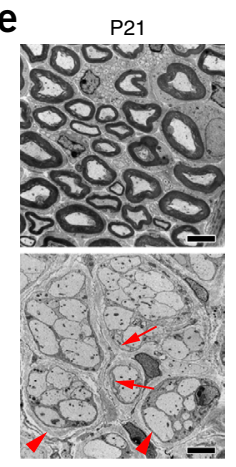

$\mathbf{f}$

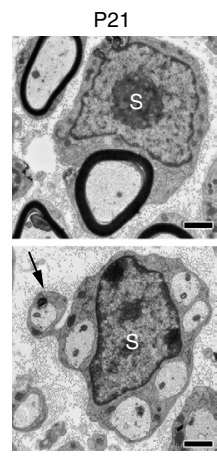

h

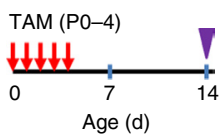

i

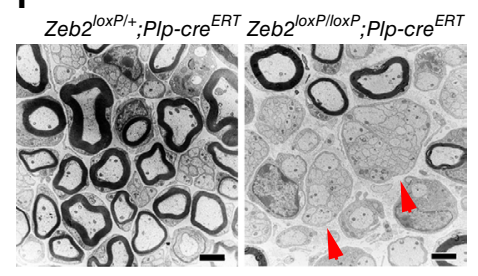

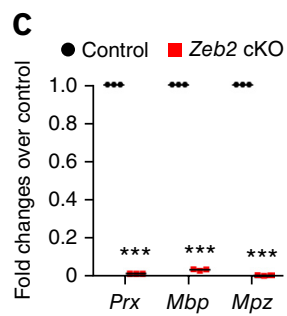

g

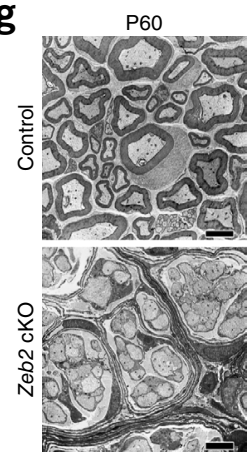

j

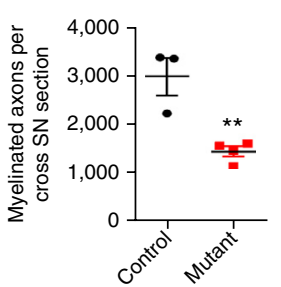


Figure 3 Zeb2 deletion in SCs inhibits SC differentiation and myelination. (a) qPCR showing levels of promyelinating transcriptional regulators in sciatic nerves of $\mathrm{P} 7$ control and Zeb2 cKO mice. Data are presented as mean \pm s.e.m. ${ }^{* *} P<0.001, n=3$ control and mutant tissues, $P_{(\text {Sox } 10)}<0.0001, t=101.4$, d.f. $=4$; $P_{(\text {Krox20 })}<0.0001, t=291.8$, d.f. $=4 ; P_{(\text {Oct6 })}$ $<0.0001, t=14,865$, d.f. $=4$; two-tailed unpaired Student's $t$-test. (b) qPCR showing the levels of negative myelination regulators in control and mutant sciatic nerves at P7. Data are presented as mean \pm s.e.m. ${ }^{*} P<0.05$, $n=3$ control and mutant tissues; $P_{(\text {Hes } 1)}=0.0308$, $t=2.617$, d.f. $=8 ; P_{(\text {Hes } 5)}=0.0081, t=4.885$, d.f. $=4 ; P_{(/ d 4)}=0.0466, t=2.846$, d.f. $=8$; $P_{(\text {Notch } 1)}=0.2181, t=1.376$, d.f. $=6$; $P_{(\text {Jag } 1)}=0.0496, t=2.784$, d.f. $=4$; two-tailed unpaired Student's $t$-test. (c) Immunolabeling of promyelinating transcription factors Sox 10 , Krox20 and Oct6 in longitudinal sections of control and Zeb2 cKO sciatic nerves

( $n=3$ animals) at P7. Scale bars, $100 \mu \mathrm{m}$. (d) Quantification of Sox $10^{+}$, Krox $20^{+}$and $\mathrm{Oct6}^{+}$cells relative to $\mathrm{DAPI}^{+}$nuclei in control and Zeb2 cKO sciatic nerves (longitudinal sections) at $\mathrm{P} 1$. Data are presented as mean \pm s.e.m. ${ }^{* *} P<0.01 ;{ }^{* *} P<0.001, n=4$ control and 6 mutant tissues for Sox 10 ;

$n=3$ control and mutant tissues for Krox20 and Oct6, $P_{(\text {Sox10 })}=0.4801, t=0.7406$, d.f. $=8$; $P_{(\text {Krox20 })}=0.0072, t=5.052$, d.f. $=4 ; P_{(\text {Oct6 })}$ $<0.0001, t=27.75$, d.f. $=4$; two-tailed unpaired Student's $t$-test. (e) Quantification of Sox $10^{+}, \mathrm{Krox}_{20} \mathrm{O}^{+}$and $\mathrm{Oct}^{+}$cells relative to $\mathrm{DAPI}^{+}$nuclei in control and Zeb2 cKO sciatic nerves at P7. Data are presented as mean \pm s.e.m. ${ }^{*} P<0.05 ;{ }^{* *} P<0.001, n=4$ control and mutant tissues for Sox $10 ; n=3$ control and mutant tissues for Krox20 and Oct6; $P_{(S o x 10)}=0.0184, t=3.120$, d.f. $=6 ; P_{(K r o x 20)}<0.0001, t=23.3$, d.f. $=4 ; P_{(0 c t 60)}<0.0001, t=16.4$, d.f. $=4$; two-tailed unpaired Student's $t$-test. (f) Immunofluorescence labeling for Ki67 in control and Zeb2 cKO sciatic nerves ( $n=3$ animals per genotype) at P28. Ki67, green; Sox10, red. Scale bars, $50 \mu \mathrm{m}$. (g) Quantification of cell proliferation by Ki67 positive nuclei in control and mutant sciatic nerves at P28. Data are presented as mean \pm s.e.m.; ${ }^{* *} P<0.01 . n=3$ control and mutant tissues; $P=0.0013, t=11.82$, d.f. $=4$, two-tailed unpaired Student's t-test. (h) Quantification of the proliferation rate by BrdU-incorporated cells per Sox10+ SC in control and mutant sciatic nerves P7. $n=3$ control and mutant tissues; Data are presented as mean \pm s.e.m.; ${ }^{*} P<0.01$. $P=0.0052, t=5.527$, d.f. $=4$; two-tailed unpaired Student's $t$-test. (i) Immunofluorescence labeling for cleaved caspase 3 in P1 control and Zeb2 cKO sciatic nerves ( $n=3$ control and mutant tissues). Cleaved caspase 3 (cl. caspase 3), white arrows; DAPI, blue. Scale bars, $100 \mu \mathrm{m}$. (j) Quantification of apoptosis by cl. caspase 3-labeled cells per $0.01 \mathrm{~mm}{ }^{2}$ in P1 control and mutant sciatic nerves. Data are presented as mean \pm s.e.m., $n=3$ control and mutant tissues, $P=0.6334, t=0.5155$, d.f. $=4$; two-tailed unpaired Student's $t$-test. (k) qPCR showing Sox10, Oct6 and Krox20 expression in rat SCs rat SCs induced to differentiate post-transfection with control and Zeb2 vectors. Data are presented as mean \pm s.e.m. ${ }^{*} P<0.05,{ }^{* *} P<0.01 ; n=3$ independent experiments, $P_{(\text {Sox10) }}=0.1826, t=1.610$, d.f. $=4 ; P_{(\text {Krox20) }}=0.0011$, $t=8.396$, d.f. $=4 ; P_{(O c t 6)}=0.04811, t=2.814$, d.f. $=4$; two-tailed unpaired Student's $t$-test. (I) qPCR showing myelin gene expression in rat SCs transfected with control and Zeb2 vectors. Data are presented as mean \pm s.e.m. ${ }^{*} P<0.05,{ }^{*} P<0.01 ; n=3$ independent experiments, $P_{(M p z)}=0.0015, t=7.775$, d.f. $=4 ; P_{(M b p)}=0.0049, t=5.626$, d.f. $=4 ; P_{(M a g)}=0.0184, t=3.843$, d.f. $=4 ; P_{(C n p)}=0.0169, t=3.942$, d.f. $=4$; two-tailed unpaired Student's $t$-test. (m) qPCR showing expression of negative regulatory genes in rat SCs transfected with control and Zeb2 vectors. Data are presented as mean \pm s.e.m. ${ }^{*} P<$ $0.05,{ }^{* *} P<0.01 ; n=3$ independent experiments; $P_{(I d 4)}=0.9738, t=0.06492$, d.f. $=4 ; P_{(\operatorname{Jag} 1)}=0.9568, t=0.05771$, d.f. $=4 ; P_{(\text {Hes })}=0.8649, t=0.1814$, d.f. $=4 ; P_{(R D p p)}=0.0105, t=4.538$, d.f. $=4 ; P_{(\text {Notch } 1)}=0.8643, t=0.1822$, d.f. $=4$; two-tailed unpaired Student's $t$-test.

observed an upregulation of Jag1, encoding a ligand for Notch, and Hes1 (Fig. 3b). In step with their transcript levels, Oct6 and Krox20 proteins were essentially absent in mutant SCs in these Zeb2 cKO sciatic nerves (Fig. 3c-e). We further investigated Zeb2 functions in SC differentiation by Zeb2 siRNA knockdown in rat SC cultures, either in proliferation or differentiation medium. The expression of Oct6, Krox20 and major myelin genes, as well as their corresponding proteins, was markedly reduced in SCs that underwent Zeb2 knockdown (Supplementary Fig. 3a-d), suggesting a cellautonomous requirement for Zeb2 in SC differentiation. In seeking to determine whether Zeb2 could regulate Oct6 directly, we identified two Zeb2 consensus-binding sites (CACCTG) (ref. 24) in the Oct6 promoter and detected genomic occupancy of Zeb2 on the Oct6 promoter in differentiating SCs but not in proliferating cells (Supplementary Fig. 3e).

The proportion of Sox $10^{+}$SCs among DAPI ${ }^{+}$cells in mutant nerves at P1 was comparable to that in control nerves (Fig. 3d), despite a slight reduction in SC number at P7 (Fig. 3e), which is likely due to the overall reduction in mature SCs. Accordingly, the SC proliferation rate revealed by proliferative marker Ki67 staining and 5-bromo-2'deoxyuridine (BrdU) incorporation appeared to increase substantially in Zeb2 mutant sciatic nerves (Fig. 3f-h). Apoptosis of mutant SCs, 
as determined by immunolabeling for cleaved caspase-3 in P1 sciatic nerves, was not significantly altered (Fig. 3i,j). This indicates that Zeb2 deletion arrests SCs at their immature stage by blocking the transition from immature SCs to $\mathrm{Oct}^{+}{ }^{+} \mathrm{Krox} 20^{+}$differentiating SCs.

Conversely, Zeb2 overexpression in SCs in vitro enhanced the induction of Oct6 and Krox20 (Fig. 3k) and myelin gene expression (Fig. 31). Notably, we detected a substantial downregulation of the Notch effector Rbpj upon enforced Zeb2 expression in SCs (Fig. 3m). Thus, our data suggests that Zeb2 controls SC myelination by priming the transcriptional program that promotes SC differentiation, while attenuating the Rbpj-mediated Notch inhibitory pathway.

\section{Zeb2 is required for SC differentiation during nerve repair}

Although Zeb2 was maintained at minimal levels in mature SCs, it was highly upregulated in SCs in regenerating sciatic nerves after injury (Fig. 4a,b). We hypothesized that Zeb2 is required for SC differentiation and ultimately remyelination during nerve repair. To test this, we employed the peripheral nerve transection model (Fig. 4a). After transection, SCs dedifferentiate, proliferate, establish a tissue bridge between the proximal and distal nerve stumps, and then guide regenerating axons across the bridge to reach the distal stump ${ }^{25}$, with subsequent SC differentiation and remyelination. To determine the role of Zeb2 in SC differentiation follow-

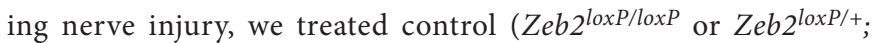
$\left.P l p 1-c r e^{E R T}\right)$ or Zeb2 $2^{\text {loxP/loxP }} ; P l p 1-c r e^{E R T}(Z e b 2$ iKO) mice at 4-6 weeks with tamoxifen before and after injury, which efficiently removed Zeb2 from transected nerves in the Zeb2 iKO mice (Fig. 4c). Sciatic nerves were removed for analysis $14 \mathrm{~d}$ after axotomy to assess SC differentiation, by which time a tissue bridge would have formed and axons guided by associated SCs as regeneration units would have proceeded to grow into the distal stump ${ }^{25,26}$. Here we focused our analysis on the regenerating site, defined as the SC-axon growth tip just proximal to the injury in the tissue bridge. In the regenerating region of Zeb2 iKO nerves, proliferation of mutant SCs $\left(\mathrm{Ki}_{67} 7^{+} \mathrm{Sox} 10^{+}\right.$cells) was indistinguishable from that of control SCs, but the number of Sox $10^{+}$SCs was significantly reduced (Fig. 4d-f). Proliferating SCs were not observed in either control or mutant contralateral sciatic nerves (data not shown). In the regenerating nerve region, mutant SCs failed to differentiate properly, as revealed by a substantial decrease in Oct6 ${ }^{+}$or Krox $20^{+}$ promyelinating SCs in mutant nerves (Fig. $4 \mathbf{g}-\mathbf{i}$ ).

We further assessed the extent of remyelination in control and Zeb2 iKO sciatic nerves by EM 8 weeks after surgery. During nerve regeneration, little, if any, radial sorting is required. In line with this, SCs lacking Zeb2 displayed normal association with axons in 1:1 ratios. While a majority of axons were remyelinated 8 weeks post injury in control nerves, Zeb2 iKO SCs were blocked at the promyelinating stage and failed to remyelinate (Fig. $\mathbf{4 j}, \mathbf{k})$. Myelin profiles in Zeb2 iKO nerves were scarce and axons that did show myelin profiles were primarily thinly myelinated. Together, our data suggest that Zeb2 is essential for SC myelination both in development and nerve regeneration.

\section{Zeb2 represses SC differentiation inhibitor Sox2}

Given severe SC myelination defects in both developing and regenerating nerves in Zeb2 mutants, we then sought to examine the expression of classic negative myelination regulators, such as Sox 2 and c-Jun ${ }^{27}$.
Figure 4 Zeb2 is required for SC differentiation during nerve repair. (a) Nerve transection scheme in control and Zeb2 iKO. Mice aged 4- to 6 weeks mice were treated with TAM via i.p. for $10 \mathrm{~d}$, followed by nerve cut injury. Mice were then given TAM for $8 \mathrm{~d}$, and nerves were analyzed at indicated days post injury (dpi). (b) Immunofluorescence labeling for Zeb2 (red) and Sox10 (green) in lesions of control sciatic nerves at $14 \mathrm{dpi}$ ( $n=3$ control and mutant tissues). SCs in the intact proximal nerves show negligible Zeb2 immunoreactivity. Scale bar, $50 \mu \mathrm{m}$. (c) Immunofluorescence labeling for Zeb2 (red) and Sox10 (green) in the regenerating site of control and Zeb2 iKO sciatic nerves 14 dpi. Arrows: Sox $10^{+} Z e b 2+$ $\mathrm{SCs}$ in control and Zeb2 iKO mutant nerves. Scale bar, $50 \mu \mathrm{m}$. (d) Immunofluorescence labeling for Ki67 (red) and Sox10 (green) in the regenerating region of control and Zeb2 iKO sciatic nerves $14 \mathrm{dpi}$ ( $n=5$ control and mutant tissues). Scale bar, $50 \mu \mathrm{m}$. (e) Quantification of Sox $10^{+}$SCs in the regenerating area in control and Zeb2 iKO sciatic nerves $14 \mathrm{dpi}$. ${ }^{*} P<0.05$; $n=5$ control and mutant tissues; $P=0.0128$,

$t=3.192$, d.f. $=8$; two-tailed unpaired Student's $t$-test. (f) Quantification of Ki67+ proliferative SCs in the regenerating area in control and Zeb2-iKO sciatic nerves 14 dpi. n.s., not significant; $n=5$ control and mutant tissues; $P=0.8308 t=0.2207$ d.f. $=8$; two-tailed unpaired Student's $t$-test. (g) Immunofluorescence labeling of Sox10 (green) and Oct6 (red, upper) or Krox20 (red, bottom) in the regenerating region of 14 dpi control and Zeb2 iKO sciatic nerves ( $n=5$ control and mutant tissues). Scale bars, $50 \mu \mathrm{m}$. (h) Quantification of the proportion of Oct6 ${ }^{+}$SCs in $14 \mathrm{dpi}$ control and Zeb2 iKO sciatic nerves in the regenerating region ( $n=4$ control and 5 mutant tissues). ${ }^{*} P<0.01 ; P=0.0087, t=3.601$, d.f. $=7$; two-tailed unpaired Student's $t$-test. (i) Quantification of the proportion of Krox20+ SCs (right) in the regenerating region of control and Zeb2 iKO sciatic nerves 14 dpi ( $n=4$ control and 5 mutant tissues). ${ }^{* * *} P<0.001 ; P=0.0004, t=6.402$, d.f. $=7$; two-tailed unpaired Student's $t$-test. (j) EM images of transverse sections of control and Zeb2 iKO 8 weeks after transection ( $n=3$ control and mutant tissues). Scale bar, $4 \mu \mathrm{m}$. (k) Quantification of the proportion of myelinated axons per field from EM images of control vs. Zeb2 iKO 8 weeks after cut injury ( $n=3$ control and mutant tissues). Data are presented as mean \pm s.e.m.; ${ }^{* *} P<0.0001 ; P<0.0001, t=37.64$, d.f. $=4$; two-tailed unpaired Student's $t$-test. For box-and-whisker plots, whiskers show minima and maxima; boxes extend from the first to the third quartiles with cross lines at the medians. 

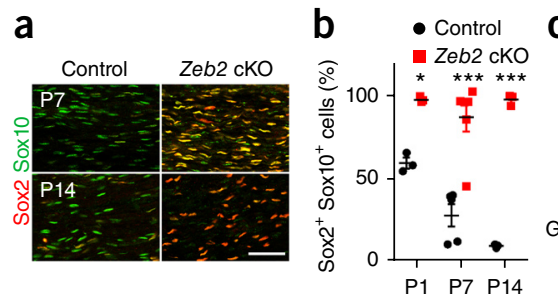

Figure 5 Zeb2 represses Sox2 activity to promote SC differentiation. (a) Immunofluorescence labeling of Sox 2 (red) and Sox 10 (green) on P7 ( $n=3$ control and mutant tissues) and P14 ( $n=4$ control and mutant tissues) control and Zeb2 cKO sciatic nerve longitudinal sections. Scale bar, $50 \mu \mathrm{m}$. (b) Quantification of Sox2+ cells relative to Sox $10^{+}$SCs in sciatic nerves of Zeb2 cKO versus controls at P1, P7 and P14. Data are presented as mean \pm s.e.m.; ${ }^{* * *} P<0.001, n=3$ control and mutant tissues for $\mathrm{P} 1 ; n=5$ control and 6 mutant tissues for P7; $n=3$ control and mutant tissues for P14. $P_{(\mathrm{P} 1)}<0.05, t=3.391$, d.f. $=17 ; P_{(\mathrm{P} 7)}<0.0001$, $t=7.079$, d.f. $=17 ; P_{(\mathrm{P} 14)}<0.0001, t=7.823$, d.f. $=17$; two-way ANOVA with Sidak's multiple comparisons test.

(c) Western blot showing persistent expression of Sox2 in sciatic nerves of Zeb2 cKO compared with controls at P14. GAPDH was the loading control. The experiment was repeated 3 times. Full-length blots are presented in Supplementary Figure 9a. (d) Immunofluorescence labeling for c-Jun (green) and Sox10 (red) in P7 control and mutant sciatic nerves ( $n=3$ animals/genotype). Scale bar, $50 \mu \mathrm{m}$. (e) Quantification of c-Jun ${ }^{+}$cells relative to $\mathrm{DAPI}^{+}$cells in sciatic nerves of Zeb2 cKO versus controls at P1 and P7. Data are presented as mean $\pm \mathrm{s}$.e.m., $n=3$ control and mutant tissues, $P_{(P 1)}=0.5188, t=0.7066$, d.f. $=4 ; P_{(P 7)}=0.2363, t=1.392$, d.f. $=4$; two-tailed unpaired Student's $t$-test. (f) Krox20 expression in SCs transfected with control (pGFP) and Sox2 (pSox2) vectors in cAMP-containing differentiation medium for $3 \mathrm{~d}$ ( $n=5$ independent experiments). Scale bar, $20 \mu \mathrm{m}$. (g) Quantification of Krox20+ among SCs transfected with pGFP and pSox2, differentiated for $3 \mathrm{~d}$ by cAMP induction. Data are presented as mean \pm s.e.m., ${ }^{* *} P<0.001, n=3$ independent experiments, $P=0.0005, t=7.922$, d.f. $=5$; two-tailed unpaired Student's $t$-test. (h) Quantification of proliferating $\mathrm{Ki} 67^{+}$cells among SCs transfected with pGFP and pSox2, cultured in neuregulin-containing proliferation medium. Data are presented as mean \pm s.e.m., $n=4$ independent experiments, $P=0.6864, t=0.4202$, d.f. $=6$; two-tailed unpaired Student's $t$-test. (i) Rat Sox2 promoter carrying the consensus Zeb2 binding sites [CACCT(g)] (red bars). TSS, transcription start site. ChIP-qPCR assays (bottom) for Zeb2 enrichment to the Zeb2 binding sites (P1-P3) in the Sox2 promoter on chromatin prepared from primary SCs exposed in proliferation medium (prolif. med.) or differentiation

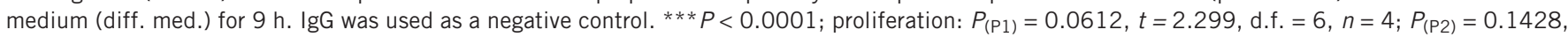
$t=1.625$, d.f. $=8, n=5 ; P_{(\mathrm{P} 3)}=0.1353, t=1.725$, d.f. $=6, n=4$; differentiation: $P_{(\mathrm{P} 1)}=0.0046, t=3.34$, d.f. $=14, n=8 ; P_{(\mathrm{P} 2)}=0.0002$, $t=5.02$, d.f. $=14, n=6 ; P_{(\mathrm{P} 3)}=0.0001, t=18.4$, d.f. $=10, n=6$; two-tailed unpaired Student's $t$-tests; $n$ : independent experiments. Whiskers show minima and maxima; boxes extend from the first to the third quartiles with cross lines at the medians. (j) qPCR showing Sox2 expression in Zeb2overexpressing SCs induced to differentiate for $18 \mathrm{~h}$, compared with vector-expressing control. Data are presented as mean $\pm \mathrm{s}$.e. $\mathrm{m}$. ${ }^{*} P<0.05 ; n=3$ independent experiments, $P=0.0211, t=3.686$, d.f. $=6$; two-tailed unpaired Student's $t$-test. (k) Rat SCs transfected with luciferase reporter driven by wild-type Sox2 promoter (-2 kb from TSS) or mutant Sox2 promoter (Zeb2 binding site deleted on P1) together with Zeb2-expressing vectors. Values represent average percentages of luciferase activity normalized to the corresponding vector expression controls from 5 independent experiments. Data are presented as mean \pm s.e.m., ${ }^{* *} P<0.01, P=0.0013, t=4.582$, d.f. $=9$; two-tailed unpaired Student's $t$-test.
At perinatal stages, in keeping with the severe reduction of myelinating SCs marked by Krox20, expression of the immature SC marker Sox2, which maintains SCs in an undifferentiated state ${ }^{27}$, persisted even at P14, when Sox 2 was rarely detectable in control nerves (Fig. 5a,b). Western blotting analysis corroborated Sox2 upregulation in postnatal mutant nerves (Fig. 5c). By contrast, c-Jun, another myelination inhibitor ${ }^{28}$, appeared normally expressed in mutant sciatic nerves compared with controls (Fig. 5d,e).

Sox 2 overexpression in SCs in vitro markedly suppressed expression of Krox20 upon induction of differentiation by dibutyryl cyclic AMP (Fig. 5f,g), though no apparent alterations of SC proliferation were detected (Fig. $\mathbf{5 h}$ ). These observations suggest that upregulation of the differentiation inhibitor Sox 2 caused by Zeb2 deletion inhibits SC differentiation.

Since Zeb2 can function as a transcriptional repressor ${ }^{24}$, we then hypothesized that it might directly target the promoter-proximal regulatory elements of Sox 2 and inhibit its expression. We identified three conserved Zeb2 consensus binding sites in the promoter region of Sox 2 within $5 \mathrm{~kb}$ upstream of the transcription start site (TSS) (Fig. 5i). Chromatin immunoprecipitation (ChIP) assays with a
Zeb2-specific antibody showed a strong enrichment of endogenous Zeb2 binding to these sites in the Sox 2 promoter region in differentiating SCs, but not to that of cells in their proliferating immature state (Fig. 5i). In contrast, no occupancy of Zeb2 to its candidate binding site in the Jun promoter was detected (Supplementary Fig. 4a). We also detected no enrichment of Zeb2 binding to Sox2 distal control regions (ChIP-PCR; Supplementary Fig. 4b). Furthermore, Zeb2 overexpression in SCs significantly reduced Sox 2 (Fig. 5j). In accord, luciferase reporter assays showed that enforced Zeb2 expression in SCs effectively repressed Sox 2 promoter activity (Fig. 5k). To address the specificity of Zeb2 action, we cotransfected the Zeb2 vector and a Sox2 promoter in which the Zeb2 binding site was mutated. Zeb2 repressive activity was significantly diminished, and luciferase reporter activity was restored to control levels (Fig. 5k). Together, our data indicate that Zeb2 directly targets the Sox 2 promoter and inhibits Sox 2 expression to promote terminal SC differentiation.

\section{Zeb2 regulates multiple SC differentiation pathways}

To identify potential additional Zeb2 targets that regulate SC differentiation, we performed RNA-seq of control and Zeb2 
cKO sciatic nerves at P7. Gene ontology analysis of differentially regulated genes indicated that the functions of downregulated genes were particularly pertinent to lipid biosynthesis, myelination and SC differentiation (Fig. 6a), whereas those of upregulated genes could be classified into categories involving cell proliferation control, adhesion and extracellular matrix organization (Fig. 6b). Consistent with our in situ hybridization and qPCR results, we detected a strong downregulation of major myelin genes and differentiation regulatory genes such as Mbp, Mag, Mpz, Krox20 and Oct6, with a concomitant increase in genes encoding myelination inhibitors, including Sox2, Jag1 and Egr1 in Zeb2 cKO sciatic nerves (Fig. 6c,d). In keeping with the dramatic sorting defect in Zeb2 cKO nerves, we detected differential expression of selected genes involved in the regulation of radial sorting, including laminin $\alpha 2$ (Lama2), Cdc42 and integrin $\alpha 6$ (Itga6) (ref. 29; Supplementary Fig. 5a,b). The RNA-seq data demonstrated an upregulation of the transforming growth factor- $\beta$ (TGF $\beta$ ) signaling genes including Tgfb2, Bmper, Smad3 and Bmp7 in Zeb2 cKO sciatic nerves (Fig. 6c); however, in contrast to the activation of bone morphogenetic protein (BMP)-receptor-activated Smads (Smad1, Smad5 and Smad8) and downregulation of Smad7 observed in oligodendrocytes lacking Zeb2 (ref. 8), we did not detect Smad activation or BMP signaling inhibition, assayed by phosphorylation of Smad1, Smad5 and Smad8 and gross Smad7 levels, respectively (Supplementary Fig. 6). These observations indicate that overall BMP-Smad signaling is not substantially altered in Zeb2 mutant SCs, suggesting that divergent mechanisms from those in CNS myelinogenesis ${ }^{8}$ are at work by Zeb2 in SCs for the control of PNS myelination.

\section{Zeb2 inhibits a Notch-Hey2 axis for SC differentiation}

We next set out to investigate other potential Zeb2-regulated signaling pathways for SC differentiation on the basis of transcriptome profiling analysis. We detected a cohort of signaling components in the Notch pathway that were significantly upregulated in Zeb2 cKO sciatic nerves (Fig. 6c). Among the upregulated genes, Hey2, a Hesrelated family basic helix-loop-helix effector of Notch ${ }^{30}$, displayed the most substantial increase in mRNA level compared to control nerves (Fig. 6d). Because Hey2 functions as a transcriptional repressor in other systems, such as in cardiac and vascular development ${ }^{30,31}$, we postulated that Hey2 might act as a blocker of SC differentiation. Hey2 transcript levels peaked at $\mathrm{P} 5$ and then rapidly declined to near zero at P15, which closely paralleled the expression profile of myelination inhibitors, such as Sox2 (Fig. 6e) 27,32. We validated the increase in Hey 2 mRNA and protein levels in Zeb2 cKO nerves by qPCR (Fig. 6f) and western blotting (Fig. 6g), respectively.

In seeking to determine whether Zeb2 could regulate Hey2 directly, we identified an evolutionarily conserved Zeb2 binding site upstream of the TSS and found that Zeb2 binding was enriched on the Hey2 promoter in differentiating SCs, but not in proliferating cells (Fig. 6h). ChIP of the distal control region of Hey2 showed no Zeb2 binding (Supplementary Fig. 4b). Moreover, enforced Zeb2 expression in SCs significantly downregulated Hey2 (Fig. 6i). Zeb2 overexpression in SCs repressed Hey2 promoter activity, and this repression was partially abolished when the Zeb2-binding site in this promoter was mutated (Fig. 6j). Overexpression of Hey2 inhibited Krox20 induction in SCs cultured under differentiation conditions (Fig. 6k,l). In contrast, the proliferative capacity of SCs

Figure 6 Zeb2 regulates the SC differentiation program and inhibits Notch effector Hey2 expression. (a,b) Gene ontology analysis of downregulated (a) or upregulated (b) genes in P7 Zeb2 cKO sciatic nerves compared with controls. Each dot in the connecting lines represents the gene count of the corresponding biological function categories. (c) Heat map showing representative differentially expressed genes and their categories in control vs. Zeb2 cKO sciatic nerves at P7; $n=2$ independent experiments. (d) qPCR analysis of genes related to SC differentiation, neural crest, Notch and Tgf $\beta$-BMP pathways in Zeb2 cKO sciatic nerves relative to controls at P7. $n=3$ independent experiments. ${ }^{*} P<0.05 ;{ }^{* *} P<0.01 ;{ }^{* *} P<0.001 ; P_{(P r x)}<0.0001$, $t=2,750$, d.f. $=4 ; P_{(M b p)}<0.0001, t=512.5$, d.f. $=4 ; P_{(\text {Sox 10) }}<0.0001, t=101.4$, d.f. $=4 ; P_{(\text {Krox } 20)}<0.0001, t=291.8$, d.f. $=4 ; P_{(0 c t 6)}<0.0001$, $t=14,865$, d.f. $=4 ; P_{(\text {Twist } 1)}=0.0003, t=11.99$, d.f. $=4 ; P_{(\text {Snail })}=0.0016, t=4.45$, d.f. $=9 ; P_{(\text {Slug })}=0.0007, t=9.39$, d.f. $=4 ; P_{(\text {Hes } 1)}=0.0974$, $t=1.962$, d.f. $=6 ; P_{(\text {Notch } 1)}=0.2186, t=1.376$, d.f. $=6 ; P_{(\mathrm{Jag} 1)}=0.0496, t=2.784$, d.f. $=4 ; P_{(\mathrm{Jag} 2)}=0.0179, t=3.879$, d.f. $=4 ; P_{(\text {Hey2 })}=0.0284$, $t=3.355$, d.f. $=4 ; P_{(\text {Tgfb2) }}=0.001, t=6.000$, d.f. $=6 ; P_{(B m p e r)}=0.0313, t=3.254$, d.f. $=4 ; P_{(B m p 7)}=0.0013, t=5.703$, d.f. $=6$; two-tailed unpaired Student's $t$-test. Whiskers show minima and maxima; boxes extend from the first to the third quartiles with cross lines at the medians. (e) qPCR analysis of Hey2 levels in mouse sciatic nerves during development ( $n=3$ control and mutant tissues for each time point). Data are presented as mean \pm s.e.m. (f) Transcript levels of Hey2 in Zeb2 cKO sciatic nerves relative to those in control nerves at P7, 15 and 30 . Data are presented as mean \pm s.e.m. ${ }^{*} P<0.05 ;{ }^{*} P<0.01 ;{ }^{* *} P<0.001, n=3$ control and mutant tissues for each time point, $P_{(\mathrm{P} 7)}=0.0136, t=5.232$, d.f. $=4 ; P_{(P 15)}$ $=0.0101, t=4.590$, d.f. $=4 ; P_{(\mathrm{P} 30)}=0.0097, t=4.641$, d.f. $=4$; two-tailed unpaired Student's $t$-test. $(\mathrm{g})$ Western blot showing Hey2 expression in control and Zeb2 cKO sciatic nerves at P14. GAPDH was the loading control. Data are from 3 independent experiments. Full-length blots are presented in Supplementary Figure 9b. (h) ChIP analysis of Zeb2 recruitment to the consensus Zeb2 binding site on the Hey2 promoter (red bar, upper panel) in primary SCs under proliferation (pro) or differentiation (diff, $9 \mathrm{~h}$ ) media. IgG IP was used as control. Data are mean \pm s.e.m.; ${ }^{* * *} P<0.001$; proliferation: $P=0.9367, t=0.08283$, d.f. $=6 n=4$; differentiation: $P=0.0005, t=10.27$, d.f. $=4 n=3$; two-tailed unpaired Student's $t$-test; $n$ : independent experiments. (i) qPCR analysis showing Hey2 gene expression in Zeb2-overexpressing SCs under differentiation media for $18 \mathrm{~h}$, compared with vector-expressing control ( ${ }^{*} P<0.05 ; n=3$ independent experiments, $P=0.0272, t=3.404$, d.f. $=4$; two-tailed unpaired Student's $t$-test). Data are presented as mean \pm s.e.m. (j) Percentages of normalized luciferase activity in rat SCs transfected with luciferase reporter driven by wild-type or mutant Hey2 promoter together with Zeb2-expressing vector. Data are presented as mean \pm s.e.m., $n=3$ independent experiments. ${ }^{* *} P<0.01$, $P=0.0025, t=6.747$, d.f. $=4$; two-tailed unpaired Student's $t$-test. (k) Krox20 expression in rat SCs transfected with control (pGFP) and Hey2expressing (pHey2) vectors under differentiation conditions for $3 \mathrm{~d}$. The experiment was repeated 3 times. Scale bars, $20 \mu \mathrm{m}$. (I) Quantification of the percentage of Krox $20^{+}$among transfected cells $\left(n=3\right.$ independent experiments). Data are presented as mean \pm s.e.m.; ${ }^{*} P<0.05 ; P=0.0411$, $t=2.733$, d.f. $=4$; two-tailed unpaired Student's $t$-test. ( $m$ ) Ki67 expression in rat SCs transfected with control (pGFP) and Hey2-expressing (pHey2) vectors for $48 \mathrm{~h}$ in proliferation medium. Scale bars, $20 \mu \mathrm{m}$. Data are from 5 independent experiments. (n) Quantification of the percentage of Ki67+ among transfected cells ( $n=5$ independent experiments). Data are presented as mean \pm s.e.m.; ${ }^{* *} P<0.01 ; P=0.0052, t=3.800$, d.f. $=8$; twotailed unpaired Student's $t$-test. (o) qPCR showing the efficiency of siRNA-mediated knockdown of Zeb2, Sox2 and Hey2. Data are presented as mean \pm s.e.m.; ${ }^{* *} P<0.001 ; n=5$ independent experiments; $P_{(\text {Zeb2) }}<0.0001, t=23.37$, d.f. $=9 ; P_{(\text {Hey } 2)}<0.0001, t=11.43$, d.f. $=8 ; P_{(\text {Sox })}<0.0001$, $t=11.47$, d.f. $=9$; two-tailed unpaired Student's $t$-test. Whiskers show minima and maxima, boxes extend from the first to the third quartiles with cross lines at the medians. (p) qPCR showing myelin-associated gene expression in rat SCs with siRNA knockdown of Zeb2 and Sox2 or Hey2 compared with single Zeb2 siRNA knockdown. Data are presented as mean \pm s.e.m.; ${ }^{* *} P<0.01$; ${ }^{* * *} P<0.001 ; n=5$ independent experiments; one-way ANOVA with Tukey's multiple comparisons test; $P_{(\text {Krox20) }}<0.001$ : scrambled vs. si-Zeb2, si-Zeb2 vs. si-Zeb2, si-Hey2; $P_{(\text {Krox20) }}<0.01$ : si-Zeb2 vs. si-Zeb2,si-Sox2; $F_{3,16}=345.8 ; P_{(M p z)}<0.001$ : scrambled vs. si-Zeb2, si-Zeb2 vs. si-Zeb2,si-Sox2; $P_{(\text {Mpz })}<0.01:$ si-Zeb2 vs. si-Zeb2,si-Hey2; $F_{3,10}=29.67 ;$

$P_{(M b p)}<0.001$ : scrambled vs. si-Zeb2, si-Zeb2 vs. si-Zeb2,si-Hey2; $P_{(M b p)}<0.05$ : si-Zeb2 vs. si-Zeb2,si-Sox2; $F_{3,10}=20.59$. 
a

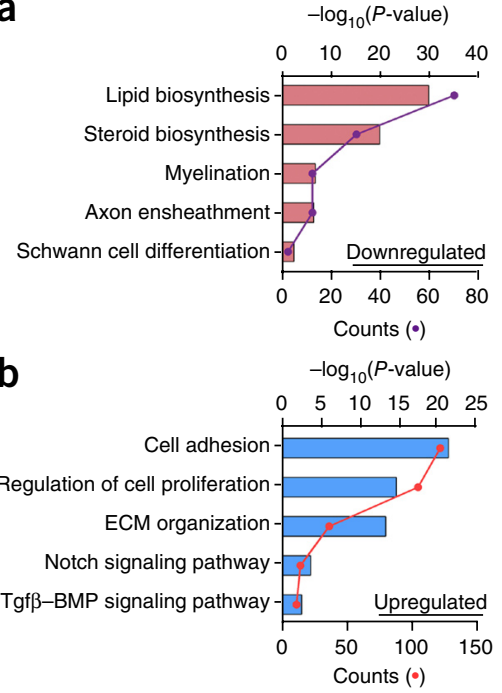

C

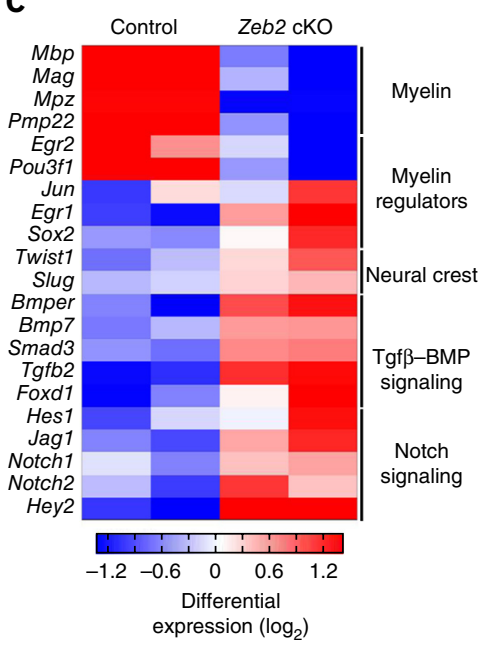

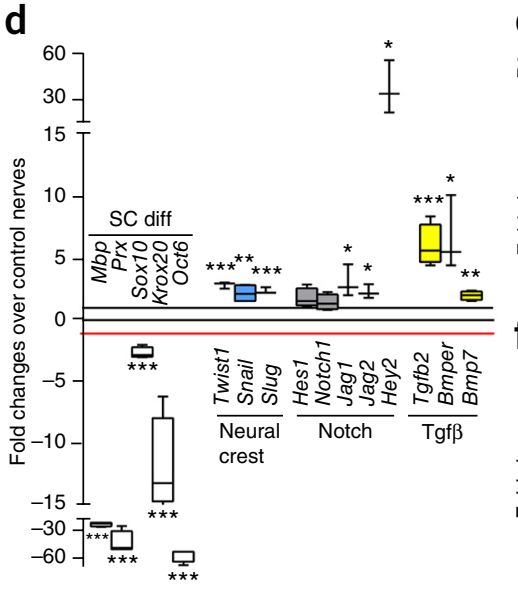

e

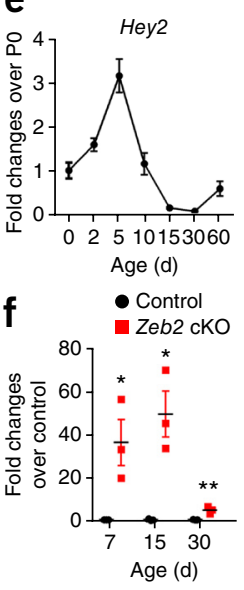

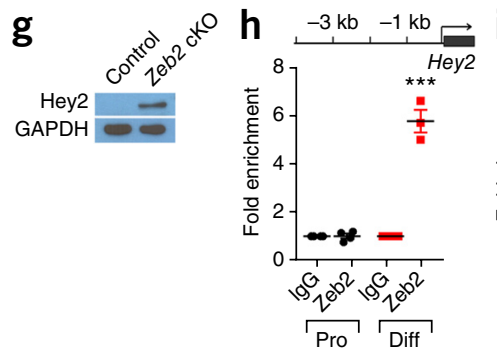

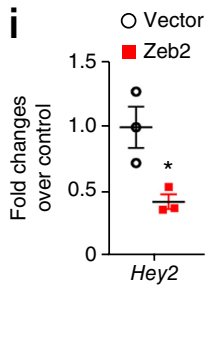

n

m

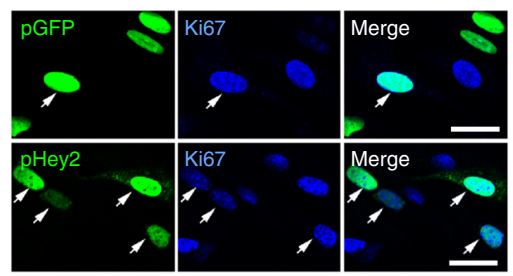

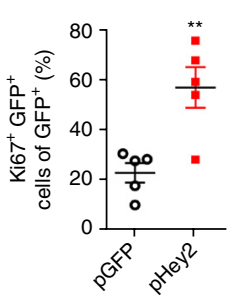

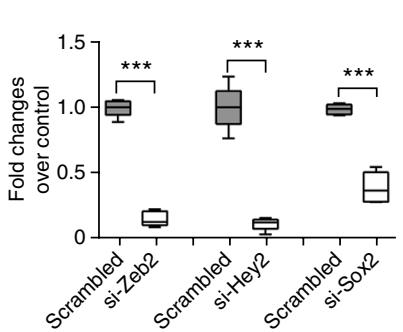

p

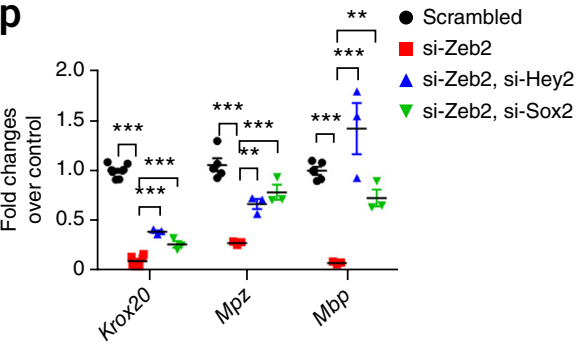

assessed by Ki67 expression with constitutive Hey2 expression was significantly higher than that of control GFP-transfected SCs (Fig. 6m,n). These observations suggest that Hey2 inhibits SC differentiation by arresting SCs in an undifferentiated state and driving their proliferation and that Zeb2 directly represses Hey2, thereby promoting SC myelination.

To further confirm that Zeb2 downregulates Hey2 or Sox2 transcription in order to promote SC differentiation, we performed sequential siRNA-mediated knockdown experiments, first by Zeb2 knockdown in SCs and then followed $24 \mathrm{~h}$ later by either knockdown of Sox 2 or Hey 2 using siRNA. Subsequent knockdown of Sox 2 or Hey2 in Zeb2-deficient SCs partially restored myelinassociated gene expression (Fig. 6o,p), indicating that Zeb2 drives SC differentiation via repression of inhibitory factor genes such as Sox 2 and Hey2.

As Notch signaling negatively controls SC myelination ${ }^{16}$, we explored the possibility that Zeb2 promotes SC differentiation and myelination by opposing a Notch-pathway-mediated differentiation block. To this end, we generated Zeb2 cKO mice carrying a Notchresponsive GFP reporter transgene (Supplementary Fig. 7a) ${ }^{33}$. There was a striking induction of Notch-dependent GFP expression in Zeb2 mutant nerves (Fig. 7a). Furthermore, overexpression of Zeb2 significantly repressed the reporter activity of a TP1 (the Epstein Barr virus terminal protein 1) promoter carrying RBP-JK (recombination signal-binding protein 1 for $\mathrm{J} \kappa)$ binding sites (Fig. $7 \mathbf{b}$ ), which are highly specific to Notch signaling activation ${ }^{34}$, suggesting that Zeb2 inhibits Notch signaling in SCs.

Notch inhibition restores differentiation of Zeb2-mutant SCs To test whether activation of Notch signaling is responsible for the differentiation failure in Zeb2-deficient SCs, we treated scrambled-siRNA and Zeb2-knockdown SCs over $4 \mathrm{~d}$ with the Notch inhibitor DAPT $(5 \mu \mathrm{M})$, which inhibits $\gamma$-secretase, a protease required for Notch1 activation $^{35}$. The addition of DAPT efficiently inhibited gene expression of Jag1 and Hey 2 in scramble-transfected SCs (Fig. 7c), whereas this treatment did not alter Zeb2 levels. In accord, SCs with Zeb2 knockdown showed increased levels of the Notch signaling genes Jag1 and Hey2. DAPT treatment effectively reduced expression of these genes (Fig. 7c) and restored expression of major myelin genes (for example, $M p z, C n p$ ) to normal levels in Zeb2-knockdown SCs (Fig. 7d). Notch inhibition by DAPT also partially rescued the otherwise perturbed induction of Oct6 in differentiating SCs with Zeb2 knockdown (Fig. 7d-f). Strikingly, treatment of Notch inhibitor LY411575 in vivo led to a robust increase in Krox $20^{+} \mathrm{SCs}$ and appearance of $\mathrm{MBP}^{+}$or 
$\mathrm{MAG}^{+}$(myelin-associated glycoprotein) myelin segments in neonatal Zeb2 cKO mice (Fig. 7g,h). Furthermore, blocking Notch signaling transduction by delivering a lentivirus expressing a dominant-negative form of the Notch coactivator Mastermind (DN-MAML) ${ }^{36}$ reduced Hey 2 or Jag1 expression and partially restored expression of Krox20 in Zeb2-knockdown SCs (Fig. 7i,j). Collectively, our data suggest that Zeb2 inhibits Notch activation, at least in part, by suppressing its downstream effector Hey2, thereby promoting differentiation of SCs from their undifferentiated state.

\section{Zeb2 recruits HDAC to antagonize differentiation inhibition}

The observation that Zeb2 could effectively suppress multiple differentiation inhibitory pathways prompted us to postulate that Zeb2 functions by forming a co-repressor complex. A previous study of the functional domain of Zeb2 identified a NuRD-interacting motif (NIM) close to the N terminus of Zeb2, which is similar to the minimal domain needed for NuRD recruitment identified in the transcription factor Sall1 (refs. 10,37). Zeb2 associates with NuRD components that include histone deacetylases HDAC1 and HDAC2 (ref. 10). HDAC1 and HDAC2 control various stages of SC development; conditional inactivation of HDAC1 and HDAC2 in the SC lineage impairs radial sorting, SC development and myelination ${ }^{38,39}$, a result that closely resembles the Zeb2 cKO phenotype. We therefore investigated the possibility that Zeb2 may interact with HDAC in the context of a NuRD co-repressor complex to suppress negative myelination regulators. Co-expression of Zeb2 together with HDAC1 or HDAC2 enhanced the repression of Hey2 and Sox2, to an extent much greater than that of Zeb2 overexpression alone, in SCs (Fig. 8a). By coimmunoprecipitation, we determined that HDAC1 and HDAC2 were present in a complex with Zeb2 (Fig. 8b). a

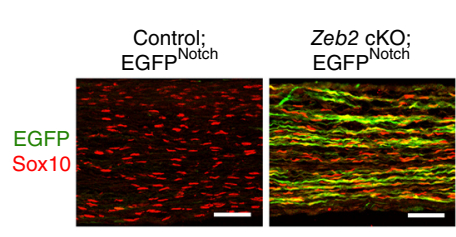

b

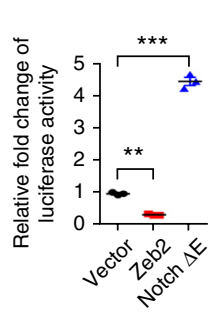

C

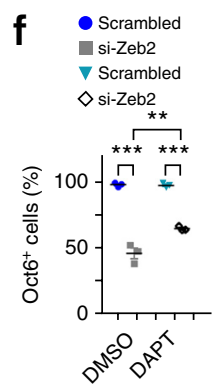

e
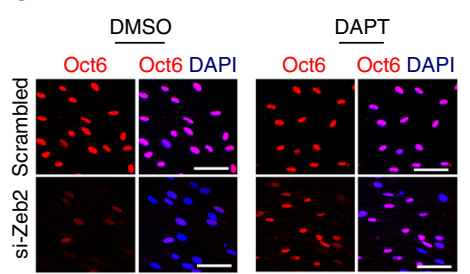
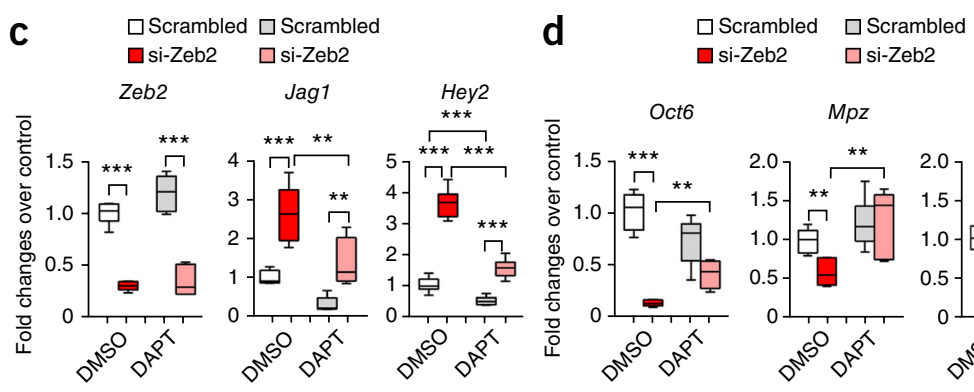

Figure 7 Zeb2 represses a Notch-Hey2 signaling axis. (a) Representative immunolabeling of GFP and Sox10 in P7 sciatic nerves of control and Zeb2 cKO mice carrying a Notch-EGFP reporter ( $n=3$ for each group). Scale bars, $50 \mu \mathrm{m}$. (b) Luciferase activity in HEK 293T cells transfected with a luciferase reporter driven by Notch-responsive TP-1 promoter (vector) and Zeb2- or Notch $\Delta \mathrm{E}$-expressing vector. Data are presented as mean \pm s.e.m., ${ }^{* *} P<0.01 ;{ }^{* * *} P<0.001 ; P_{(Z e b 2)}<0.001 ; P_{(T p 1)}<0.0001 ; F_{2,6}=890.1 ; n=3$ independent experiments; one-way ANOVA with Tukey's multiple comparison tests. (c) qPCR showing Jag1 and Hey2 expression in Zeb2 knockdown in SCs treated with $5 \mu$ M DAPT over 4 d. ${ }^{*} P<0.05$; $* * P<0.01$; $* * * P<0.001$, one-way ANOVA with Tukey's multiple comparisons test; Zeb2: $P_{(\text {DMSO })}<0.0001, P_{(\text {DAPT })}<0.0001 ; F_{3,20}=79.78 ;$ Jag1: $P_{(\text {DMSO })}<0.001, P_{(\text {DAPT })}<0.01 ; P_{(\text {si-Zeb2, DMSO vs. DAPT })}<0.01 ; F_{3,19}=22.75 ;$ Hey2: $P_{(\text {DMSO })}<0.0001, P_{(\text {DAPT })}<0.0001 ; P_{(\text {scrambled, DMSO vs. DAPT })}$ $<0.05 ; P_{(\text {si-Zeb2, DMSO vs. DAPT) }}<0.0001 ; F_{3,20}=119.5$; data are from 6 independent experiments. (d) qPCR showing Oct6, Mpz and Cnp expression in Zeb2-knockdown SCs treated with DAPT. ${ }^{*} P<0.01$; ${ }^{* * *} P<0.001$; one-way ANOVA with Tukey's multiple comparison's test; Oct6: $P_{\text {(DMSO) }}<0.0001$; $P_{(\mathrm{si}-\text { Zeb2, DMSO vs. DAPT })}<0.01 ; F_{3,18}=33.29 ; M p z: P_{(\mathrm{DMSO})}<0.01 ; P_{(\mathrm{si}-\text { Zeb2, DMso vs. DAPT })}<0.01 ; F_{3,19}=6.745 ;$ Cnp: $P_{(\mathrm{DMSO})}<0.05 ; P_{(\mathrm{si}-\text { Zeb2 }}$, DMSO vs. DAPT) $<0.001 ; F_{3,19}=10.16$; data are from 5 independent experiments. For $\mathbf{c}$ and $\mathbf{d}$, whiskers show minima and maxima; boxes extend from the first to the third quartiles with cross lines at the medians. (e) Immunostaining of Oct6 (red) in scrambled or si-Zeb2-knockdown SCs treated with DMSO or DAPT for $4 \mathrm{~d}$ in differentiation medium. DAPI, blue. The experiment was repeated 3 times. Scale bars, $50 \mu \mathrm{m}$. (f) Quantification of Oct6-expressing cells among scrambled or si-Zeb2-knockdown SCs treated with DMSO or DAPT for $4 \mathrm{~d}$ in differentiation medium. Data are presented as mean \pm s.e.m.; ${ }^{*} P<0.01 ;{ }^{* *} P<0.001$; one-way ANOVA with Tukey's multiple comparison's test; $P_{(\text {DMSO })}<0.001, P($ DAPT $)<0.001$; $P_{(\text {si-Zeb2, DMSO vs. DAPT) }}<0.01 ; F_{3,8}=134.6$. Data are from 3 independent experiments. (g) Immunostaining of Krox20, MBP or MAG in sciatic $n$ erves of Zeb2 cKO mice treated with vehicle or Notch-inhibitor LY411575 from P2 to P5 and killed on P8. Arrows indicate $\mathrm{MBP}^{+}$or MAG+ myelin segments. Data are from 3 individual mice from each group. Scale bars, $50 \mu \mathrm{m}$. (h) Quantification of Krox $20^{+}$nuclei in Zeb2 cKO sciatic nerves treated with vehicle or LY411575. Data are presented as mean \pm s.e.m.; $n=3$ individual mice from each group; ${ }^{*} P<0.05 ; P=0.0135, t=4.221$, d.f. $=4$; two-tailed unpaired Student's t-test. (i) qPCR showing Jag1 and Hey2 expression in Zeb2-knockdown SCs transduced with GFP- or DNMAMLGFP-expressing lentivirus for $48 \mathrm{~h}$ in proliferation medium. Data are presented as mean \pm s.e.m.; ${ }^{* *} P<0.01$; ${ }^{* *} P<0.001$; one-way ANOVA with Tukey's multiple comparison test, Jag1: $P_{\text {(scrambled GFP vs. si-Zeb2 GFP) }}<0.01, P_{\text {(si-Zeb2 GFP vs. si-Zeb2 DN-MAmL GFP) }}<0.01 ; F_{2,6}=18.91 ; H e y 2: P_{(\text {scrambled GFP }}$

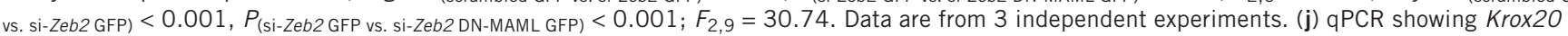
levels in Zeb2-knockdown SCs transduced with GFP- or DNMAML-GFP-expressing lentivirus. Data are presented as mean \pm s.e.m.; ${ }^{* * *} P<0.001$; one-way ANOVA with Tukey's multiple comparison test $P_{\text {(scrambled GFP vs. si-Zeb2 GFP })}<0.0001, P_{\text {(si-Zeb2 GFP vs. si-Zeb2 DN-MAML GFP })}<0.0001 ; F_{2,16}=244.8$. Data are from 5 independent experiments. 
We identified a human ZEB2 variant [c.64A>G; Arg22Gly] carrying a single residue $Z E B 2$ mutation (R22G) in an individual with mild

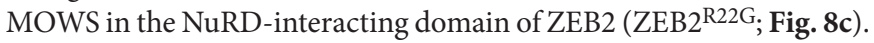
Strikingly, we found that $\mathrm{ZEB} 22^{\mathrm{R} 22 \mathrm{G}}$ was sufficient to abolish HDAC1 and HDAC2 recruitment to the ZEB2 complex (Fig. 8d). Moreover, this missense mutation in ZEB2 markedly disrupted ZEB2 interaction with other NuRD subunits as determined by coimmunoprecipitation, whereas CtBP (C-terminal-binding protein 1) binding was unaffected (Fig. 8e and Supplementary Fig. 7b). We further confirmed the endogenous interaction of Zeb2 with NuRD complex components, including HDAC1, HDAC2 and Mi2/Chd4, in rat SCs (Fig. 8f). Most notably, exogenous expression of $\mathrm{ZEB} 2^{\mathrm{R}} 22 \mathrm{G}$ markedly diminished the induction of Oct6 and Krox20 in SCs (Fig. 8g-i). However, overexpressing a Zeb2 mutant protein with deletion of CtBP-interacting g

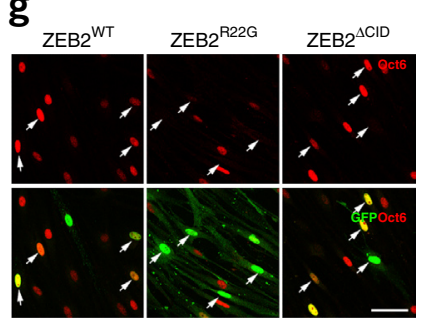

a

b

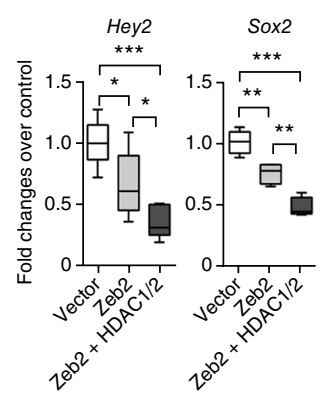

c

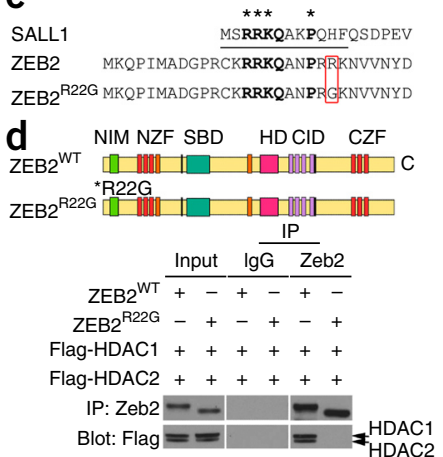

e

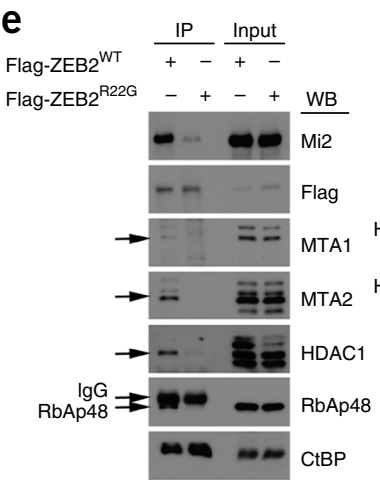

\section{$\mathbf{f}$}

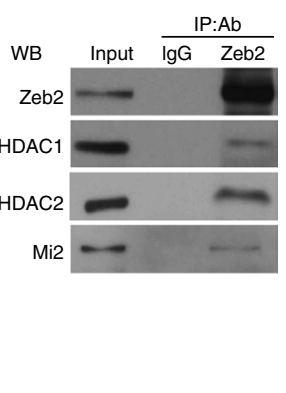

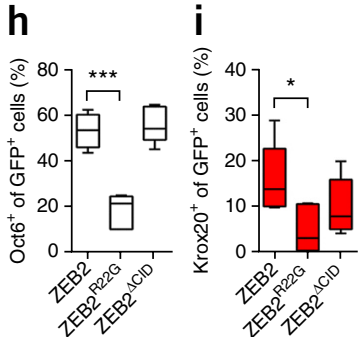

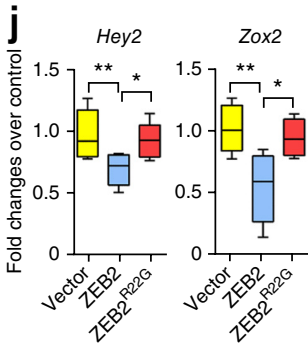

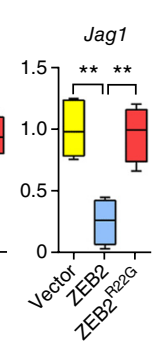
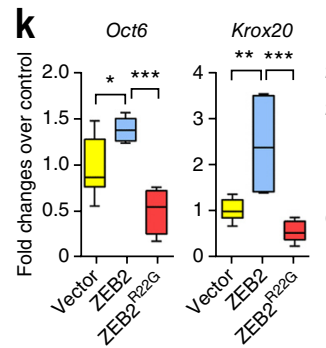

Cnp

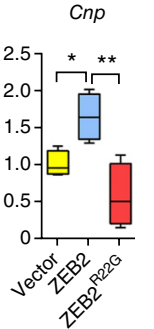

Figure 8 Zeb2 associates with HDAC1 and HDAC2 to repress SC differentiation inhibitor expression. (a) qPCR showing the fold change in Hey2 and Sox2 expression in SCs overexpressing Zeb2 or coexpressing Zeb2, HDAC1 and HDAC2 relative to vector-expressing cells. ${ }^{*} P<0.05 ;{ }^{* *} P<0.01 ;{ }^{* *} P<0.001$; one-way ANOVA with Tukey's multiple comparison tests; Hey2: $P_{\text {(vector vs. Zeb2) }}<0.05, P_{\text {(Zeb2 vs. Zeb2 }+ \text { Hdac1/2) }}<0.05 ; P_{(\text {vector vs. Zeb2 }+ \text { Hdac1/2) }}<0.0001$; $F_{2,20}=15.72$; Sox2: $P_{(\text {vector vs. Zeb2) }}<0.001, P_{(Z e b 2 \text { vs. Zeb2 }+H \text { dac 1/2) }}<0.001 ; P_{(\text {vector vs. Zeb2 }+ \text { Hdac1/2) }}<0.0001$; $F_{2,10}=40.59$. Data are from 3 independent experiments. (b) Co-immunoprecipitation with Flag-tagged HDAC1 or HDAC2 in HEK 293T cells cotransfected with Myc-tagged Zeb2. IP, immunoprecipitation; GAPDH, loading control. Full-length blots are presented in Supplementary Figure 9c. The experiment was repeated 3 times. (c) Alignment of SALL1, human wild-type and mutant ZEB2 ${ }^{\mathrm{R} 22 \mathrm{G}}$ in NURD binding domain (underline). Amino acids in bold are critical for NuRD binding. The red box highlights the amino acid change in the ZEB2R22G mutant. (d) Top: schematic domains

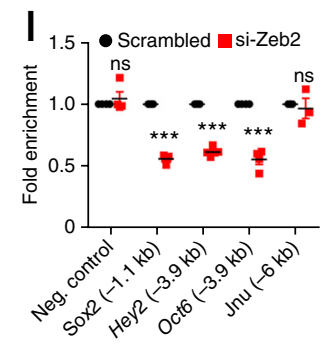
in Zeb2 ${ }^{W T}$ and Zeb2R22G proteins. NZF and CZF, N- and C-terminal zinc finger domains, respectively; NIM, NuRD interacting motif; SBD, Smad-binding domain; HD, homeodomain-like domain; CID, CtBP interacting domain. Bottom: coimmunoprecipitation of Zeb2 in HEK 293T cells transfected with Flag-tagged HDAC1 and HDAC2 together with Zeb2 or Zeb2R22G expressing vectors. IgG was used as the IP control. The experiment was repeated 3 times. Full-length blots are presented in Supplementary Figure 9d. (e) Western blot showing coimmunoprecipitation of endogenous NuRD subunits with ZEB2WT but not ZEB2R22G in HEK293T cells transiently expressing Flag-ZEB2WT or Flag-ZEB2R22G. The experiment was repeated 3 times. Full-length blots are presented in Supplementary Figure 10b. (f) Western blot showing coimmunoprecipitation with an antibody to Zeb2 and control lgG in rat SCs using antibodies to HDAC1, HDAC2 and Mi-2. The experiment was repeated 3 times. Full-length blots are presented in Supplementary Figure 10a. (g) Immunolabeling of Oct6 or Krox20 in rat SCs transfected with Zeb2WT, Zeb2R22G or Zeb2 ${ }^{\Delta C I D}$ vectors carrying a GFP reporter under differentiation conditions for $48 \mathrm{~h}$. Arrows, transfected cells. Scale bar, $50 \mu \mathrm{m}$. Data are from 5 independent experiments. (h) Quantification of the proportion of Oct6+ cells among transfected GFP+ cells ( $n=5$ independent experiments). ${ }^{* * *} P<0.001$. One-way ANOVA with Tukey's multiple comparison tests;

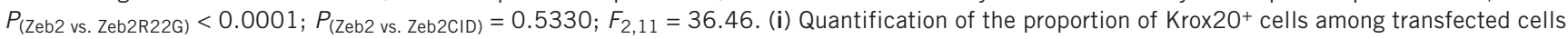

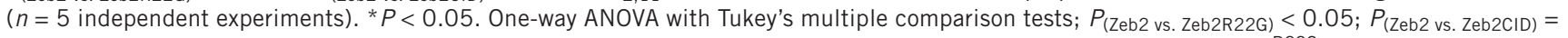
0.2225; $F_{2,12}=3.497$. (j) qPCR analysis of Zeb2 targets, Jag1, Hey2 and Sox2 in rat SCs transfected with Zeb2 or Zeb2R22G mutant expression vectors. ${ }^{*} P<0.05 ;{ }^{*} P<0.01$; one-way ANOVA with Tukey's multiple comparisons test; Hey2: $P_{\text {(vector vs. Zeb2) }}<0.01, P_{(\text {Zeb2 vs. Zeb2R22G) }}<0.05$; $F_{2,21}=6.884 ; n=7$ independent experiments; Jag1: $P_{\text {(vector vs. Zeb2) }}<0.01, P_{\text {(Zeb2 vs. Zeb2R22G) }}<0.01 ; F_{2,10}=16.24 ; n=4$ independent experiments; Sox2: $P_{\text {(vector vs. Zeb2) }}<0.01, P_{\text {(Zeb2 vs. Zeb2R22G) }}<0.05 ; F_{2,13}=7.584 ; n=5$ independent experiments. (k) qPCR analysis of myelin-related genes Oct6, Krox20 and Cnp in rat SCs transfected with Zeb2 or Zeb2R22G mutant expression vectors. ${ }^{*} P<0.05 ;{ }^{* *} P<0.01$; ${ }^{* * *} P<0.01$; one-way ANOVA with Tukey's multiple comparisons tests; Oct6: $P_{\text {(vector vs. Zeb2) }}<0.05, P_{\text {(Zeb2 vs. Zeb2R22G) }}<0.001 ; F_{2,15}=20.24 ; n=5$ independent experiments; Krox20: $P_{\text {(vector vs. Zeb2) }}$ $<0.01, P_{\text {(Zeb2 vs. Zeb2R22G) }}<0.001 ; F_{2,14}=12.31 ; n=6$ independent experiments; $C n p: P_{\text {(vector vs. Zeb2) }}<0.05, P_{(\text {Zeb2 vs. Zeb2R22G) }}<0.01 ; F_{2,9}=11.25 ;$ $n=4$ independent experiments. (I) HDAC1 occupancy by ChIP-PCR in rat SCs knocked down with control or Zeb2 siRNA on the promoters of Zeb2 target genes. Chromatin was from SCs induced to differentiate by cAMP-containing medium for 9 h. IgG IP was used as a negative control. Data are mean \pm s.e.m.; ${ }_{* * *} P<0.001 ; n=$ at least 3 independent experiments; $P_{(\text {negative control })}=0.4683, t=0.7741$, d.f. $\left.=6 ; P_{(\text {Sox2, }} 1.1 \mathrm{~kb}\right)<0.0001, t=32.85$, d.f. $=8$; $P_{(\text {Hey2, } 3.9 \mathrm{~kb})}<0.0001, t=24.63$, d.f. $=8 ; P_{(0 c t 6,3.9 \mathrm{~kb})}<0.0001, t=11.26$, d.f. $=6 ; P_{(\text {Jun }, 6 \mathrm{~kb})}=0.6954, t=0.4209$, d.f. $=4$; two-tailed unpaired Student's $t$-test. For box-and-whisker plots, whiskers show minima and maxima; boxes extend from the first to the third quartiles with cross lines at the medians. 
domains (Zeb2 $\left.2^{\Delta \mathrm{CID}}\right)^{40}$ did not alter induction of either Oct6 (Fig. 8g-h) or Krox20 (Fig. 8i) in SCs. Accordingly, enforced ZEB2 ${ }^{\mathrm{R} 22 \mathrm{G}}$ expression in SCs also lost the capacity to repress Zeb2 target genes, such as Hey2, Sox2 and Jag1, or to activate the transcription of the myelin-related genes Oct6, Krox20 and Cnp (Fig. 8j-k).

To determine whether recruitment of HDAC1 and HDAC2 by Zeb2 is required for target expression, we performed ChIP-qPCR in Zeb2-deficient SCs. The reduction of Zeb2 in SCs significantly compromised the enrichment of HDAC1 on the Zeb2-consensus binding regions in the promoters of Zeb2 target genes, such as Sox2, Oct6 and Hey2, in Zeb2-knockdown SCs (Fig. 81). In contrast, HDAC1 occupancy on the promoter of a non-Zeb2 target, Jun, in Zeb2-knockdown SCs was comparable with that in IgG controls (Fig. 81). Similar results were also obtained for HDAC2 recruitment (data not shown). This suggests that the Zeb2-HDAC1-HDAC2 complex is required for the regulation of target gene transcription in SCs. Thus, our findings indicate that Zeb2 represses SC differentiation inhibitors by associating with the HDAC1-HDAC2-NuRD co-repressor complex to promote SC myelination.

\section{DISCUSSION}

In this study, we provided in vivo genetic evidence for the critical function of Zeb2 in SC precursor differentiation and peripheral nerve myelination, as well as remyelination during nerve regeneration, consistent with the observations by Quintes et al. ${ }^{41}$ in this issue. We establish Zeb2 as a key regulatory switch that controls the timing for the onset of normal SC differentiation and remyelination after injury. Furthermore, our findings reveal that Zeb2 cooperates with a HDAC1-HDAC2-NuRD chromatin-remodeling complex as a repressor targeting a network of inhibitory pathways, including Sox 2 and Notch signaling, to drive SC differentiation, in conjunction with acting as a transcriptional activator of other target genes such as Oct6 (ref. 42). Moreover, we identified a Zeb2-modulated Notch-Hey2 signaling axis that blocks SC differentiation. Inhibition of Notch signaling partially rescues the SC differentiation defect caused by Zeb2 deficiency. Thus, Zeb2 regulates SC differentiation and myelination at least in part through de-repression of Notch signaling (Supplementary Fig. 7c,d), pointing to the importance of HDAC1-HDAC2-NuRDdependent ZEB2 activity in MOWS-associated neuropathies.

\section{Zeb2 recruits HDAC1/2-NuRD to control SC differentiation}

Delineation of differentially expressed genes by transcriptome analysis reveals that Zeb2 modulates inhibitory pathways, namely Sox2 effects and Notch-Hey2 signaling, during SC differentiation. Whether Zeb2 regulation of these individual inhibitory signals exists in parallel, and whether Zeb2 mediates its actions through other unidentified cofactors of these or other pathways to control SC myelination, would require further investigation. Nonetheless, we find that Zeb2 engages the HDAC1/2-NuRD complex to exert repressive activity on myelination inhibitory factors. Several studies have reported radial sorting and myelination impairment in mouse mutants with $\mathrm{SC}$-specific inactivation of $\mathrm{NuRD}$ subunits or associated factors including Mi-2/Chd 4 and HDAC1/2 $2^{38,39,43}$ as well as interacting partners such as NAB proteins ${ }^{32,44}$. Co-expression of Zeb2, HDAC1 and HDAC2 in SCs further augments repression in expression of inhibitory genes Sox 2 and Hey2. Remarkably, a single missense mutation, Zeb2 $2^{\mathrm{R} 22 \mathrm{G}}$, in the Zeb2 N-terminal NuRD-interacting domain completely abolishes its association with the HDAC1/2-NuRD and inhibits SC differentiation, likely due to inefficient repression of the aforementioned differentiation-inhibitory signals. Thus, by interacting with the HDAC1/2-NuRD complex ${ }^{45}$, Zeb2 may mediate its repressive activity to fine-tune transcription of the genes that negatively impact myelination. Taken together, our data indicate that Zeb2 acts as a key transcriptional repressor that effectively extinguishes multiple inhibitory pathways to direct the onset of SC differentiation and initiation of myelination.

\section{A Notch-Hey2 axis for inhibiting SC differentiation}

Notch signaling regulates multiple stages of SC development, from promoting SC formation and proliferation to negatively regulating myelination ${ }^{16}$. Strikingly, in the absence of Zeb2, Notch signaling is turned on constitutively in sciatic nerves, exerting a negative regulatory effect on SC differentiation and myelination. We observed a substantial increase of proliferating immature SCs in Zeb2 mutants, which is consistent with the notion that activation of Notch signaling leads to increased SC proliferation ${ }^{16}$. The transcriptome profiling analysis further identified Hey2, a transcriptional repressor downstream of Notch ${ }^{46}$, as a Zeb2 target that suppresses Krox20 expression and blocks SC differentiation.

Nonetheless, unlike the sustained block of SC maturation in Zeb2 cKO nerves, genetically enforced Notch (i.e., NCID) expression in SCs does not completely prevent the initiation of myelinogenesis ${ }^{16}$. Therefore, we cannot rule out the possibility that Hey2 regulation of SC development may also be independent of canonical Notch signaling, as reported in other contexts ${ }^{47,48}$. If such is the case, Zeb2regulated Hey2 would introduce an additional layer of repression to the already existing Notch inhibition on SC myelination.

Zeb2-deficient SCs exhibit persistent upregulation of Sox2 and a substantial increase in Sox $2^{+}$immature SCs in peripheral nerves. Sox 2 has been shown to act downstream of Notch signaling in certain contexts. For instance, Notch activation induces Sox 2 in sensory progenitors of the inner ear ${ }^{49}$. Elevation of Sox2 levels maintains SCs in the undifferentiated state by suppressing expression of SC differentiation markers such as Krox $20^{32}$. Given that Hey2 overexpression inhibits Krox20 and increases the proliferation of immature SCs (Fig. 6k-n), Notch-Hey2 activation may induce or maintain Sox 2 expression to inhibit SC differentiation.

\section{Distinct mechanisms of Zeb2 in CNS and PNS myelination}

Recent studies of Zeb2 revealed that it activates Smad7 and inhibits the action of activated BMP-Smads on genes that would otherwise be inhibitory for oligodendrocyte myelinogenesis and hence promotes myelinogenesis in the $\mathrm{CNS}^{8}$. In contrast, the present study shows that Zeb2 does not appear to control SC differentiation via regulation of BMP-Smad signaling or Smad7 expression in the PNS, suggesting that Zeb2 controls SC myelination via a different mechanism. Here we find that Zeb2 directs SC differentiation by a disinhibition mechanism via antagonizing Notch-Hey2 signaling and Sox2 in the PNS, which was not observed in the $\mathrm{CNS}^{8}$. Furthermore, we found that in the PNS Zeb2, through recruitment of the HDAC1/2-NuRD, negatively regulates Sox 2 and the Notch effector Hey2 to guard against the blockade of SC myelination mediated by these inhibitors.

In contrast to substantial downregulation of Sox10 in oligodendrocytes of the Zeb2 mutant spinal cord ${ }^{8}$, Zeb2 does not seem to regulate Sox10 (Fig. 3d,k). However, we cannot exclude the possibility that Sox10 and Zeb2 coordinate to drive SC differentiation, similarly to the cooperative role they play in the enteric nervous system $^{50}$. Alternatively, we observed a preferential recruitment of Zeb2 to the Oct6 promoter when SCs begin to differentiate (Supplementary Fig. 3e). Given the downregulation of key promyelination regulators Oct6 and Krox20 in Zeb2-ablated sciatic nerves, our data suggests that Zeb2 may function as a dual-level switch 
control of SC differentiation through inactivating multiple differentiation inhibitors, including Notch signaling and Sox2, while promoting pro-myelinating factors Oct6 and Krox20 during SC development.

Zeb2 inactivation in SCs during nerve repair inhibits SC redifferentiation and thus remyelination, supporting the crucial role of Zeb2 in myelin regeneration in the PNS. However, we did not detect any difference in Sox 2 or Hey 2 mRNA expression in the mutant injured nerves $7 \mathrm{~d}$ post-transection, at which time regenerating mouse nerves would have reached the midpoint of axonal regrowth, as compared to the control injured sciatic nerves (Supplementary Fig. 8). Thus, we cannot rule out the possibility that Zeb2 may utilize other mechanisms after injury for SC regeneration and remyelination.

Deficient radial axonal sorting and myelination caused by Zeb2 loss could severely disrupt nerve conduction and, consequently, motor function in Zeb2 mutant mice, paralleling motor disabilities and delayed walking in humans with MOWS. The phenotypic variation between humans with haplo-insufficient mutations could be attributed to a differential sensitivity to gene dosage across species or dominant-negative effects of human mutant proteins. Strikingly, we find that the $\mathrm{ZEB} 2{ }^{\mathrm{R} 22 \mathrm{G}}$ detected in a MOWS patient failed to associate with HDAC1/2-NuRD and blocked SC differentiation, suggesting that developmental defects caused by ZEB2 mutations affecting a functional domain may contribute to neuropathies and motor dysfunction seen in these patients. Given that ZEB2-mediated functions are vital for SC development and myelination, it may serve as a pivotal hub of the myelination regulatory network. Augmenting HDAC1/2-NuRD-dependent ZEB2 activity and inhibiting Notch signaling could potentially provide a novel therapeutic means of reversing adverse neuropathies seen in people with MOWS.

\section{METHODS}

Methods and any associated references are available in the online version of the paper.

\section{Accession codes GEO: GSE74381.}

Note: Any Supplementary Information and Source Data files are available in the online version of the paper.

\section{ACKNOWLEDGMENTS}

The authors thank X. Chen and Z. Ma for technical support and initial observation of Zeb2 mutants. We thank A. Rauch for discussing ZEB2 missense mutations in MOWS patients, J. Svaren and E. Hurlock for suggestions and G. Verstappen and L. van Grunsven for initial study of Zeb2 ${ }^{\mathrm{R} 22 \mathrm{G}_{-}}$NuRD interactions. We are grateful to K.-A. Nave for communications of unpublished data. We also thank R. Kopan (University of Cincinnati) and A.J. Capiobianco (University of Miami) for the TP-1 reporter and lentiviral DN-MAML constructs and to J.W. Schneider and E.N. Olson (University of Texas Southwestern Medical Center)) for Notch-GFP reporter mice and Flag-HRT2/Hey2 vectors. This study was funded in part by grants from the US National Institute of Health R01NS072427 and R01NS075243 to Q.R.L., R01NS062796 to J.R.C. and R01AR064551 to M.P.J., and from the National Multiple Sclerosis Society (NMSS-4727) to Q.R.L. L.M.N.W. was supported by an NMSS Postdoctoral Fellowship (FA 2045A1/T). The work was also supported by Belspo grant IAP7-07 DevRepair, the Research Council of KU Leuven (GOA11/012), FWO-V (G.0782.14), the type 3 large-infrastructure support InfraMouse by the Hercules Foundation (ZW09-03) and Erasmus MC start-up funds, to D.H.

\section{AUTHOR CONTRIBUTIONS}

L.M.N.W. and Q.R.L. designed the experiments. L.M.N.W. carried out the studies. J.W., A.C. and L.Z. assisted with coimmunoprecipitation biochemical experiments for HDAC-NuRD and BMP-Smads. C. Zhao assisted with ChIP experiments. H.W. assisted with Notch-TP1 reporter assays. C. Zweier provided ZEB2 variant identification data. Z.F. assisted with heat hypersensitivity experiments. M.P.J. assisted with CMAP recordings. B.G.A., P.M., A.Z. and J.R.C. provided input and data interpretation. D.H. provided mice with the loxP-flanked Zeb2 allele and input on the study. L.M.N.W. and Q.R.L. wrote the manuscript.

\section{COMPETING FINANCIAL INTERESTS}

The authors declare no competing financial interests.

Reprints and permissions information is available online at http://www.nature.com/ reprints/index.html.

1. Pereira, J.A., Lebrun-Julien, F. \& Suter, U. Molecular mechanisms regulating myelination in the peripheral nervous system. Trends Neurosci. 35, 123-134 (2012).

2. Nave, K.A. \& Werner, H.B. Myelination of the nervous system: mechanisms and functions. Annu. Rev. Cell Dev. Biol. 30, 503-533 (2014).

3. Salzer, J.L. Schwann cell myelination. Cold Spring Harb. Perspect. Biol. 7, a020529 (2015).

4. Dastot-Le Moal, F. et al. ZFHX1B mutations in patients with Mowat-Wilson syndrome Hum. Mutat. 28, 313-321 (2007).

5. Mowat, D.R., Wilson, M.J. \& Goossens, M. Mowat-Wilson syndrome. J. Med. Genet 40, 305-310 (2003).

6. Nagaya, M., Kato, J., Niimi, N., Tanaka, S. \& Wakamatsu, N. Clinical features of a form of Hirschsprung's disease caused by a novel genetic abnormality. J. Pediatr. Surg. 37, 1117-1122 (2002).

7. McKinsey, G.L. et al. Dlx1\&2-dependent expression of Zfhx1b (Sip1, Zeb2) regulates the fate switch between cortical and striatal interneurons. Neuron 77, 83-98 (2013).

8. Weng, Q. et al. Dual-mode modulation of Smad signaling by Smad-interacting protein Sip1 is required for myelination in the central nervous system. Neuron 73 , 713-728 (2012).

9. van den Berghe, V. et al. Directed migration of cortical interneurons depends on the cell-autonomous action of Sip1. Neuron 77, 70-82 (2013).

10. Verstappen, G. et al. Atypical Mowat-Wilson patient confirms the importance of the novel association between ZFHX1B/SIP1 and NuRD corepressor complex. Hum. Mol. Genet. 17, 1175-1183 (2008).

11. Michailov, G.V. et al. Axonal neuregulin-1 regulates myelin sheath thickness. Science 304, 700-703 (2004).

12. Taveggia, C. et al. Neuregulin-1 type III determines the ensheathment fate of axons. Neuron 47, 681-694 (2005).

13. Feltri, M.L. \& Wrabetz, L. Laminins and their receptors in Schwann cells and hereditary neuropathies. J. Peripher. Nerv. Syst. 10, 128-143 (2005).

14. Nodari, A. et al. Alpha6beta4 integrin and dystroglycan cooperate to stabilize the myelin sheath. J. Neurosci. 28, 6714-6719 (2008).

15. Monk, K.R. et al. A G protein-coupled receptor is essential for Schwann cells to initiate myelination. Science 325, 1402-1405 (2009).

16. Woodhoo, A. et al. Notch controls embryonic Schwann cell differentiation, postnatal myelination and adult plasticity. Nat. Neurosci. 12, 839-847 (2009).

17. Jaegle, M. et al. The POU proteins Brn-2 and Oct-6 share important functions in Schwann cell development. Genes Dev. 17, 1380-1391 (2003).

18. Topilko, P. et al. Krox-20 controls myelination in the peripheral nervous system. Nature 371, 796-799 (1994).

19. He, Y. et al. Yy1 as a molecular link between neuregulin and transcriptional modulation of peripheral myelination. Nat. Neurosci. 13, 1472-1480 (2010).

20. Svaren, J. \& Meijer, D. The molecular machinery of myelin gene transcription in Schwann cells. Glia 56, 1541-1551 (2008).

21. Higashi, Y. et al. Generation of the floxed allele of the SIP1 (Smad-interacting protein 1) gene for Cre-mediated conditional knockout in the mouse. Genesis 32 82-84 (2002).

22. Douglas, W.W. \& Ritchie, J.M. Mammalian nonmyelinated nerve fibers. Physiol. Rev. 42, 297-334 (1962).

23. Doerflinger, N.H., Macklin, W.B. \& Popko, B. Inducible site-specific recombination in myelinating cells. Genesis 35, 63-72 (2003).

24. Verschueren, K. et al. SIP1, a novel zinc finger/homeodomain repressor, interacts with Smad proteins and binds to $5^{\prime}$-CACCT sequences in candidate target genes. J. Biol. Chem. 274, 20489-20498 (1999).

25. Jessen, K.R., Mirsky, R. \& Lloyd, A.C. Schwann cells: development and role in nerve repair. Cold Spring Harb. Perspect. Biol. 7, a020487 (2015).

26. Parrinello, S. et al. EphB signaling directs peripheral nerve regeneration through Sox2-dependent Schwann cell sorting. Cell 143, 145-155 (2010).

27. Jessen, K.R. \& Mirsky, R. The origin and development of glial cells in peripheral nerves. Nat. Rev. Neurosci. 6, 671-682 (2005).

28. Parkinson, D.B. et al. c-Jun is a negative regulator of myelination. J. Cell Biol. 181, 625-637 (2008).

29. Feltri, M.L., Poitelon, Y. \& Previtali, S.C. How Schwann cells sort axons: new concepts. Neuroscientist 22, 252-265 (2016).

30. Fischer, A., Schumacher, N., Maier, M., Sendtner, M. \& Gessler, M. The Notch target genes Hey 1 and Hey2 are required for embryonic vascular development. Genes Dev. 18, 901-911 (2004).

31. Xin, M. et al. Essential roles of the bHLH transcription factor Hrt2 in repression of atrial gene expression and maintenance of postnatal cardiac function. Proc. Natl. Acad. Sci. USA 104, 7975-7980 (2007).

32. Le, N. et al. Analysis of congenital hypomyelinating Egr2Lo/Lo nerves identifies Sox2 as an inhibitor of Schwann cell differentiation and myelination. Proc. Natl. Acad. Sci. USA 102, 2596-2601 (2005).

33. Mizutani, K., Yoon, K., Dang, L., Tokunaga, A. \& Gaiano, N. Differential Notch signalling distinguishes neural stem cells from intermediate progenitors. Nature 449, 351-355 (2007). 
34. Souilhol, C. et al. Nas transgenic mouse line allows visualization of Notch pathway activity in vivo. Genesis 44, 277-286 (2006).

35. De Strooper, B. et al. A presenilin-1-dependent gamma-secretase-like protease mediates release of Notch intracellular domain. Nature 398, 518-522 (1999).

36. Pinnix, C.C. et al. Active Notch 1 confers a transformed phenotype to primary human melanocytes. Cancer Res. 69, 5312-5320 (2009).

37. Lauberth, S.M. \& Rauchman, M. A conserved 12-amino acid motif in Sall1 recruits the nucleosome remodeling and deacetylase corepressor complex. J. Biol. Chem. 281, 23922-23931 (2006).

38. Chen, Y. et al. HDAC-mediated deacetylation of NF-KB is critical for Schwann cell myelination. Nat. Neurosci. 14, 437-441 (2011).

39. Jacob, C. et al. HDAC1 and HDAC2 control the transcriptional program of myelination and the survival of Schwann cells. Nat. Neurosci. 14, 429-436 (2011)

40. van Grunsven, L.A. et al. XSip1 neuralizing activity involves the co-repressor CtBP and occurs through BMP dependent and independent mechanisms. Dev. Biol. 306, 34-49 (2007).

41. Quintes, S. et al. Zeb2 is essential for Schwann cell differentiation, myelination and nerve repair. Nat. Neurosci. http://dx.doi.org/10.1038/nn.4321 (2016).

42. Conidi, A. et al. Few Smad proteins and many Smad-interacting proteins yield multiple functions and action modes in TGF $\beta / B M P$ signaling in vivo. Cytokine Growth Factor Rev. 22, 287-300 (2011).
43. Hung, H., Kohnken, R. \& Svaren, J. The nucleosome remodeling and deacetylase chromatin remodeling (NuRD) complex is required for peripheral nerve myelination. J. Neurosci. 32, 1517-1527 (2012).

44. Desmazières, A., Decker, L., Vallat, J.M., Charnay, P. \& Gilardi-Hebenstreit, P. Disruption of Krox20-Nab interaction in the mouse leads to peripheral neuropathy with biphasic evolution. J. Neurosci. 28, 5891-5900 (2008).

45. Reynolds, N., O'Shaughnessy, A. \& Hendrich, B. Transcriptional repressors: multifaceted regulators of gene expression. Development 140, 505-512 (2013).

46. Fischer, A. et al. Hey basic helix-loop-helix transcription factors are repressors of GATA4 and GATA6 and restrict expression of the GATA target gene ANF in fetal hearts. Mol. Cell. Biol. 25, 8960-8970 (2005).

47. Doetzlhofer, A. et al. Hey2 regulation by FGF provides a Notch-independent mechanism for maintaining pillar cell fate in the organ of Corti. Dev. Cell 16 58-69 (2009).

48. Benito-Gonzalez, A. \& Doetzlhofer, A. Hey1 and Hey2 control the spatial and temporal pattern of mammalian auditory hair cell differentiation downstream of Hedgehog signaling. J. Neurosci. 34, 12865-12876 (2014).

49. Liu, Z., Owen, T., Fang, J., Srinivasan, R.S. \& Zuo, J. In vivo Notch reactivation in differentiating cochlear hair cells induces Sox2 and Prox1 expression but does not disrupt hair cell maturation. Dev. Dyn. 241, 684-696 (2012).

50. Stanchina, L., Van de Putte, T., Goossens, M., Huylebroeck, D. \& Bondurand, N. Genetic interaction between Sox10 and Zfhx1b during enteric nervous system development. Dev. Biol. 341, 416-428 (2010). 


\section{ONLINE METHODS}

Animals. Zeb2 $2^{\text {loxP/loxP }}$ mice ${ }^{21}$ were crossed with $D h h$-cre ${ }^{17}$ mice to obtain Zeb2 $2^{\text {loxP/+}} ; D$ hh $-c r e^{+/-}$mice, which were then bred with Zeb2 loxP/loxP mice to produce control (Zeb2 $\left.2^{l o x P /+} ; D h h-c r e^{+/-}\right)$and Zeb2 cKO offspring (Zeb2 $2^{\text {loxP/loxP }}$; $\left.D h h-\mathrm{cre}^{+/-}\right)$. Littermates Zeb2 $2^{\text {loxP/loxP }}$ or Zeb2 $2^{\text {loxP/+}} ; \mathrm{Dhh}-\mathrm{cre}^{+/-}$mice were used as controls. Transgenic Notch reporter (TNR) mice were obtained from the Jackson

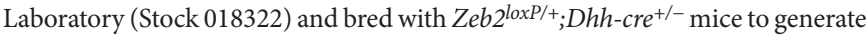
Zeb2 $2^{\text {loxP/loxP }} ;$ Dhh-cre ${ }^{+/-} ;$Notch-GFP mice. Recombination, perinatally or after sciatic nerve transection, was achieved by crossing Zeb2 $2^{\text {loxP } / l o x P}$ mice with the inducible Cre recombinase Cre-ERT2 under the control of the Plp1 promoter $\left(P l p 1-c r e^{E R T}\right)$ followed by tamoxifen injection ${ }^{23}$. Zeb2 $2^{l o x P} /$ loxP $;$ Plp1-cre $e^{E R T}$ mice were then bred to mice containing a Cre-responsive Rosa26 loxP-flanked stop tdTomato reporter allele. Animals of either sex were used in the study and littermates were used as controls unless otherwise indicated. The mouse strains used in this study were generated and maintained on a mixed C57Bl/6;129Sv background and housed in a vivarium with a 12 -h light/dark cycle. No more than 4 adult mice were housed in the same cage per IACUC regulations at CCHMC. All animal experiments were conducted in mice of both genders. All animal use and studies were approved by the Institutional Animal Care and Use Committee at Cincinnati Children's Hospital, USA.

Isolation, growth and differentiation of primary rat SCs. Rat SCs from sciatic nerves of newborn rats (1-2 d old) were isolated as described previously ${ }^{51}$. SCs were grown routinely in DMEM/10\% FBS (Life Technologies), supplemented with $10 \mathrm{ng} / \mathrm{ml}$ Neuregulin 1 (Nrg1; R\&D Systems) and $5 \mu \mathrm{M}$ forskolin (Sigma) plus L-glutamine and penicillin/streptomycin, hereafter termed SC proliferation medium. Cells between passages 2 and 6 were used in all experiments. $>95 \%$ SC purity was achieved, assessed by Sox 10 and $S 100 \beta$ staining. To initiate differentiation, SCs were washed 4 times with DMEM and then cultured in differentiation medium containing DMEM/0.5\% FBS and $1 \mathrm{mM}$ dibutyryl cyclic AMP (Sigma) with L-glutamine and penicillin/streptomycin, for the length of time indicated in the text, depending on the assays used. All tissue culture containers and coverslips were coated with $50 \mu \mathrm{g} / \mathrm{ml}$ poly-L-lysine (Sigma) in PBS for at least $30 \mathrm{~min}$ at room temperature and then rinsed in distilled water. Purified rat SCs seeded on coverslips were fixed in $4 \%$ paraformaldehyde (PFA) for $20 \mathrm{~min}$ and washed in $1 \times$ PBS 4 times before immunofluorescence staining.

Immunostaining, In situ hybridization and electron microscopy. The sciatic nerves of mice at defined ages were dissected and fixed for $45 \mathrm{~min}$ in $4 \%$ PFA, embedded in OCT, cryoprotected in $25 \%$ sucrose and sectioned at $9 \mu \mathrm{m}$ as longitudinal sections. For BrdU pulse labeling, animals at P1 were injected subcu-

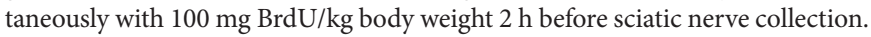
For immunostaining, we used antibodies to Zeb2 (Santa Cruz Biotechnology, sc-48789), Sox10 (goat, Santa Cruz Biotechnology, sc-17342; rabbit, Millipore; AB5727), Oct6 (goat; Santa Cruz Biotechnology, sc-11661), Krox20 (rabbit; Covance, PRB-236P), MBP (goat; Santa Cruz Biotechnology, sc-13914), Sox2 (goat; Santa Cruz Biotechnology, sc-17320), c-Jun (rabbit; Epitomics, 1254-1), Hey2 (rabbit; Proteintech, 10597-1-AP); Ki67 (rabbit; Thermo Scientific, RM9106), BrdU (rat; Abcam, ab6326), cleaved caspase 3 (rabbit; Cell Signaling, \#9661), S100 $\beta$ (mouse; Sigma, SAB1402349); GFP (rabbit; Life Technologies, A11122); c-MYC (mouse; Santa Cruz Biotechnology, sc-40), Flag (mouse; Cell Signaling, \#8146) and HA-tag (Upstate, 50-171-890). Secondary antibodies conjugated to $\mathrm{Cy} 2, \mathrm{Cy} 3$ or $\mathrm{Cy} 5$ were from Jackson ImmunoResearch Laboratories catalog numbers 705-165-147, 705-225-147, 711-225-152, 711-165-152, 711175-152, 715-165-150 and 712-165-150. All images were acquired using a Zeiss LSM 510 or Nikon $\mathrm{C}^{+}$confocal microscope. RNA in situ hybridization was performed using digoxigenin-labeled riboprobes as described previously ${ }^{52}$. The probes used were murine Mbp and Mpz. Detailed protocols are available upon request. For electron microscopy, mice were perfused with 4\% PFA, $2.5 \%$ glutaraldehyde in $0.1 \mathrm{M}$ sodium cacodylate buffer, $\mathrm{pH}$ 7.2. Sciatic nerves were dissected and fixed in the same fixative solution overnight. Nerves were rinsed in PBS, postfixed in $1 \%$ OsO4 in PBS for $1 \mathrm{~h}$, dehydrated in graded ethanol, infiltrated with propylene oxide and embedded in Epon. Semi-thin sections were stained with toluidine blue, and thin sections were stained with lead citrate.

Transient transfections and luciferase assays. For plasmid transfections, rat SCs were transfected with expression vectors using Lipofectamine 3000 (Life
Technologies) per the manufacturer's protocol and assayed for immunocytochemistry or qPCR analysis. pHA-Sox2 was purchased from Addgene.

For siRNA knockdown in SCs, we used Lipofectamine RNAiMAX (Life Technologies) per the manufacturer's instructions. SCs were induced to differentiate after $48 \mathrm{~h}$ of transfection for $24 \mathrm{~h}$ before harvesting for immunocytochemistry or qPCR analysis. For sequential siRNA knockdown, SCs in proliferation medium was transfected with Zeb2-specific siRNA for $24 \mathrm{~h}$ before Sox 2 or Hey2 siRNA transfection. Triple knockdown was performed in the order of Zeb2, Hey2 and Sox2 siRNA, each separated by a 24-h interval. Zeb2 siRNA: SASI_Rn01_00044097 (GCAAGAAAUGUAUUGGUUU); SASI Rn01_00044098 (GUAUGCAUGUGACUUA UGU); SASI_Rn02_00203739 (CAGAUGAACCUCUGAAUUU). Sox2 siRNA: CCACCUACAGCAUGUC CUA[dT][dT]; Hey2 siRNA: GGAUCGAAUAAAUAACAGU[dT][dT] and CCAUGCAGAUUCUGCUCUU[dT][dT].

For DAPT (Sigma) treatment, $48 \mathrm{~h}$ after transfection with scramble or Zeb2 siRNA, SCs on coverslips or in 12-well culture dishes were induced to differentiate with $1 \mathrm{mM}$ dbcAMP and treated with DMSO or $5 \mu \mathrm{M}$ DAPT for $4 \mathrm{~d}$ and then assayed for immunocytochemistry or qRT-PCR analysis. Proliferation was assessed by immunostaining for Ki67 in Sox $10^{+}$SCs seeded on PLL-coated coverslips for $2 \mathrm{~d}$. Differentiation was assessed by immunostaining for Oct6 or Krox20. Multiple images were taken from each coverslip to obtain representative images from all areas of the coverslip, and at least 400 cells/coverslip were counted using ImageJ (NIH). For quantitation, $\mathrm{GFP}^{+}$or fusion tag-expressing cells were counted, and the percentage of $\mathrm{GFP}^{+} /$fusion$\mathrm{tag}^{+}$cells that expressed the protein of interest was determined. At least $100 \mathrm{GFP}^{+}$ or fusion-tag ${ }^{+}$cells/coverslip were counted.

For reporter assays, purified rat SCs were transfected with pGL3luciferase reporters containing inserts from $2 \mathrm{~kb}$ upstream of Sox 2 TSS or $3.9 \mathrm{~kb}$ upstream of Hey2 TSS and with expression vectors using Lipofectamine 3000 (Life Technologies) per the manufacturer's protocol, and were assayed $48 \mathrm{~h}$ posttransfection for luciferase activity using a Promega luciferase assay kit according to the manufacturer's instructions. The $\mathrm{pSV}$ - $\beta$-galactosidase control vector was included to control for variable transfection efficiencies between independent experiments $(n \geq 3)$. Mutant Sox 2 promoter luciferase reporter was generated by mutating a Zeb2 consensus binding site ( $\mathrm{P} 1 ;-1.1 \mathrm{~kb}$ from TSS; Fig. $5 \mathbf{i})$ into an EcoRI site, and mutant Hey2 promoter luciferase reporter was generated by mutating a Zeb2 binding site into an EcoRI site.

Western blotting and coimmunoprecipitation. For western blotting, the perineurium and epineurium were removed from sciatic nerves before snap-freezing and storage at $-80{ }^{\circ} \mathrm{C}$. Sciatic nerves and rat SCs were lysed in RIPA buffer, containing protease and phosphatase inhibitors. Western blot analysis was performed as described previously ${ }^{38}$. GAPDH was used as an input control. For immunoprecipitation, HEK 293T cells cultured in $10 \%$ FBS/DMEM were transfected with expression vectors using PolyJet (Signagen) for $48 \mathrm{~h}$. Cells were lysed in NP-40 buffer ( $170 \mathrm{mM} \mathrm{NaCl}, 10 \mathrm{mM}$ EDTA, $50 \mathrm{mM}$ Tris pH 7.4, $50 \mathrm{mM} \mathrm{NaF}$ and 0.5\% NP-40) supplemented with protease inhibitor cocktail and phosphatase inhibitors (Roche Diagnostics Inc.). A total of $300 \mu \mathrm{g}$ of cell lysate proteins were incubated with $2 \mu \mathrm{g}$ IgG or appropriate antibodies and immunoprecipitated using Protein A/G beads (Santa Cruz Biotechnology, sc-2003). Western blotting was performed using chemiluminescence with the ECL kit (Pierce). The antibodies used were anti-Zeb2 (Millipore, ABT-332), anti-Flag (rabbit \#14793 or mouse \#8146, Cell Signaling), anti-Myc (Santa Cruz, sc-40). For coimmunoprecipitation of endogenous NuRD subunits, extracts of HEK 293T cells transiently expressing Flag-tagged ZEB2 ${ }^{\mathrm{WT}}$ or Flag-tagged ZEB2 ${ }^{\mathrm{R} 22 \mathrm{G}}$ were precipitated in a Flag-dependent manner and eluted using 3XFlag peptides according to Verstappen et all ${ }^{10}$. Co-immunoprecipitation experiments showing endogenous interaction between Zeb2 and HDAC1/2 were performed by incubating protein lysates from purified rat SCs with anti-Zeb2 antibody followed by immunoprecipitation using Protein A/G beads and detection of HDAC1/2 using HDAC1/2-specific antibodies. Antibodies used were HDAC1 (Santa Cruz, sc-7872), HDAC2 (Abcam \#7029), Mi2 (Santa Cruz, sc-11378), MTA-1 (Santa Cruz, sc-9446), MTA-2 (Santa Cruz, sc-28731), RbAp48 (Abcam, 38185) and CtBP (Sigma, C8741). Secondary antibodies conjugated to HRP were from Jackson ImmunoResearch Laboratories. catalog numbers 111035-144 and 111-035-003. 
RNA-seq and data analysis. RNA from P7 control and Zeb2 cKO sciatic nerves were extracted using TRIZOL (Life Technologies) followed by purification using an RNeasy Mini Kit (Qiagen). RNA-seq libraries were prepared using Illumina RNA-Seq Preparation Kit and sequenced by HiSeq 2000 sequencer. RNA-seq reads were mapped using TopHat with default settings (http://ccb.jhu. edu/software/tophat/index.shtml). TopHat output data were then analyzed by Cufflinks to (1) calculate FPKM values for known transcripts in mouse genome reference and (2) test the changes of gene expression between Zeb2 cKO and control. Gene ontology (GO) analysis was performed using The Database for Annotation, Visualization and Integrated Discovery (DAVID; http://david.abcc. ncifcrf.gov). The heat map was generated based on $\log _{2}[\mathrm{FPKM}]$ by AltAnalyze ${ }^{53,54}$ with normalization of rows relative to row mean.

ChIP assays. ChIP assays were performed as described with minor modifications ${ }^{38,55}$. Briefly, purified rat SCs grown in proliferation and differentiation ( $9 \mathrm{~h}$ in $1 \mathrm{mM}$ cAMP-containing medium conditions; 20 million cells) were fixed for $10 \mathrm{~min}$ at room temperature with $1 \%$ formaldehyde-containing medium. Nuclei were pelleted and sonicated in sonication buffer $(10 \mathrm{mM}$ Tris- $\mathrm{HCl}(\mathrm{pH}$ 8.0), $1 \mathrm{mM}$ EDTA, $0.5 \mathrm{mM}$ EGTA and protease inhibitor cocktail). Sonicated chromatin $(\sim 300 \mu \mathrm{g})$ was used for immunoprecipitation by incubation with IgG or rabbit anti-Zeb2 antibody (H-260; Santa Cruz Biotechnology sc-48789; $4 \mu \mathrm{g}$ ) overnight at $4{ }^{\circ} \mathrm{C}$. We kept $10 \%$ of the chromatin used for each ChIP reaction as input DNA. Pre-rinsed protein A/G plus agarose beads ( $50 \mu \mathrm{l})$ were added to each ChIP reaction and incubated for $1 \mathrm{~h}$ at $4{ }^{\circ} \mathrm{C}$. The beads were then incubated in $200 \mu \mathrm{l}$ elution buffer at $65^{\circ} \mathrm{C}$ for $20 \mathrm{~min}$ to elute immunoprecipitated materials. For ChIP, SCs at 70-90\% confluency were transfected with control or Zeb2 siRNA for $48 \mathrm{~h}$ using Lipofectamine RNAiMAX per the manufacturer's instructions and induced to differentiate for $9 \mathrm{~h}$ with $1 \mathrm{mM}$ cAMP. ChIP was performed using anti-HDAC1 (Active Motif \#40967) and HDAC2 antibody (Abcam \#7029). Real-time PCR was performed using quantitative SYBR green PCR mix (Bio-Rad). The relative fold-enrichments were determined by $2^{-\Delta C T}$ methods as described ${ }^{38}$. Samples were normalized to input chromatin. Primers used for ChIP-qPCR analysis:

Sox2 proximal promoter (P1): forward ATTGGTGGAGGAGGGAGTTA; reverse TATGGCTGACTGTAGAGCTAGG;

Sox2 proximal promoter (P2): forward CTGCACCTGCACCTCTG; reverse CAGCGTTCCCAGGATGG;

Sox2 proximal promoter (P3): forward CTCCACCCGTGTTTCCTAAAT; reverse CTTCTGCCCTCTAAGTCACAAA;

Hey 2 proximal promoter ( $-3.9 \mathrm{~kb}$ from TSS): forward: GCTCACAATA GCCTGTAGTT; reverse: AAGTCAGTCTCTGTCCTGGT.

Oct6 proximal promoter ( $-0.8 \mathrm{~kb}$ from TSS): forward: TCCTAGAGCTCG CATCTCC; reverse: ATCGGCTTGGGCTTCAC.

Oct6 proximal promoter ( $-3.9 \mathrm{~kb}$ from TSS): forward: GTGTCCGT ATGTCTTCGAGATG; reverse: ACACGGTGCTATTCACACAA.

Jun proximal promoter ( $-6 \mathrm{~kb}$ from TSS): forward: CCCAAGAC CTGTGTGAGAAT; reverse: CTCACAGTTTGATTGGCTGAAA.

Distal control region ChIP-PCR: Sox2 (+25 kb TSS): forward GAACAGATG AGTGTTGCCTTTAG; reverse ACTCCCTGGACCAAACATAC;

Hey2 (+20 kb TSS): forward GGAAACTGTATACGGCCTCTG; reverse GATCTGCATCGCCCTTTCT;

Oct6 (+18 kb TSS): forward GTAGAGGAAAGCAGAGGAAAGG; reverse GAATCCCATAGCCAGACATGAT.

RNA isolation and quantitative real-time PCR. RNA from purified rat SCs or control and Zeb2 cKO mouse sciatic nerves was extracted using TRIZOL (Life Technologies). cDNA was synthesized from $1 \mu \mathrm{g}$ RNA using iScript Reverse Transcription Supermix (BioRad) according to the manufacturer's instructions. qPCR was performed using quantitative SYBR green PCR mix (BioRad) as previously described ${ }^{56}$. PCR primer sequences are available upon request.

RNA-seq and data analysis. RNA-seq libraries were prepared using Illumina RNA-Seq Preparation Kit and sequenced by a HiSeq 2000 sequencer. All RNASeq data were aligned to $\mathrm{mm} 9$ using TopHat ${ }^{57}$ with default settings. We used Cuff-diff ${ }^{58}$ to (1) estimate FPKM values for known transcripts and (2) analyze differentially expressed transcripts. In all differential expression tests, a difference was considered significant if the q value was less than 0.05 (Cuff-diff default).
Heat map of gene expression was generated using R language (http://www.rproject.org). GO-analysis of gene expression changes was performed using Gene Set Enrichment (GSEA, http://www.broadinstitute.org/gsea/index.jsp). We used ToppCluster (https://toppcluster.chmc.org/) to construct the network of genes belonging to over-represented GO-term categories.

Tamoxifen injections. We used Tamoxifen (Sigma) dissolved to a stock concentration of $20 \mathrm{mg} / \mathrm{ml}$ in a vehicle of ethanol and sunflower seed oil $(1: 9 \mathrm{v} / \mathrm{v})$. For perinatal tamoxifen injections, tamoxifen stock was injected i.p. at $2 \mathrm{mg} / 100$ $\mu \mathrm{l}$ for 5 consecutive $\mathrm{d}$ to lactating mothers, thus administering tamoxifen to pups, beginning at $\mathrm{P} 0$. Sciatic nerves of pups were analyzed on P14 for electron microscopy. For adult tamoxifen treatment, $100 \mu \mathrm{l}(2 \mathrm{mg} / 100 \mu \mathrm{l})$ was administered by i.p. injection once daily for 5 consecutive d to $4-6$ week-old mice. Mice were treated again for $5 \mathrm{~d}$ after a 2 -d rest period. Control mice were treated identically.

In vivo drug treatment. Control and Zeb2 cKO were injected on $\mathrm{P} 2, \mathrm{P} 3, \mathrm{P} 5$ and P7 with the Notch signaling ( $\gamma$-secretase) inhibitor LY411575 (Selleckchem) at $0.30 \mathrm{mg} \mathrm{kg}^{-1}$ per day. Sciatic nerves were fixed in $4 \%$ PFA on P8 for immunostaining. LY411575 was diluted in a mixture of corn oil and EtOH $(95: 5 \mathrm{v} / \mathrm{v})$. Vehicle was corn oil and EtOH (95:5 v/v).

Generation and transduction of lentivirus. Lentivirus was produced by transfection of DN-MAML lentivirus construct (MAML1 1-305 subcloned into BamHI and XhoI sites of PLU-ires-GFP mod vector, a kind gift from A.J. Capobianco) into HEK 293T cells with the packaging vectors psPAX2 and pMD2.G using Polyjet in vitro DNA transfection reagent (Sigmagen) according to the instructions of the manufacturer. The supernatant was collected $72 \mathrm{~h}$ after transfection and frozen in liquid nitrogen. A titer of $10^{7} / \mu \mathrm{l}$ was achieved. SCs previously transfected with control or Zeb2 siRNA for $24 \mathrm{~h}$ were infected with control GFP lentivirus or DNMAML-GFP lentivirus in the presence of $4 \mu \mathrm{g} / \mathrm{ml}$ Polybrene for $48 \mathrm{~h}$ before harvesting for RNA.

ZEB2 variant identification. The novel ZEB2 variant c. 64A $>\mathrm{G}$ [p.(Arg22Gly)] was identified by targeted diagnostic sequencing of the coding exons of ZEB2 in a patient with a facial gestalt reminiscent of MOWS (OMIM 235730). The patient had mild manifestation of MOWS phenotype. This mutation was not detected in any other patients and is not contained in the ExAC database. This variant is atypical since missense mutations in ZEB2 are very rare in MOWS; usually truncating mutations are found. Due to the young age (4 years old) of the patient, peripheral nerve-related phenotyping was clinically impossible to test for, and therefore we could not assess the peripheral nerve related phenotype.

Sciatic nerve transection surgeries. Sciatic nerve transection was performed $10 \mathrm{~d}$ after the last tamoxifen injection in 6-8 week-old mice. Under general anesthesia after injection of a mixture of ketamine $(90 \mathrm{mg} / \mathrm{kg})$ and xylazine $(10 \mathrm{mg} / \mathrm{kg})$, right sciatic nerves were exposed and transected at the midthigh level. Exposed left sciatic nerves were used as uncut controls. Mice were treated with tamoxifen for an additional $8 \mathrm{~d}$ following injury. Nerves were collected $14 \mathrm{~d}$ after surgery and processed for immunohistochemistry.

Compound muscle action potential recording. To analyze nerve conduction and motor unit function, single CMAPs were recorded in vivo from the lateral gastrocnemius muscles of wild-type littermate controls or Zeb2 $\mathrm{cKO}$ mice during electrical stimulation of the sciatic nerve under sodium pentobarbital anesthesia $(50 \mathrm{mg} / \mathrm{kg}$, i.p.). The lateral gastrocnemius muscle was first exposed from the knee to approximately $6 \mathrm{~mm}$ above the ankle. A small opening was then made in the overlying fascia to facilitate implantation of the recording wires into the muscles. Next, the sciatic nerve was exposed near the biceps femoris muscles. Polymidecoated steel recording wires (California Fine Wire Inc. stainless steel 304: size = 0.0092 and insulated with polyimide; material \#100192) were then implanted into the lateral gastrocnemius muscles and reference wires were inserted under the nearby skin. To induce electrical activation of the nerve in vivo, a concentric bipolar stimulating electrode was placed on the sciatic nerve. CMAPs were amplified and obtained using a Micro 1401 data acquisition unit and analyzed using Spike2 software (Cambridge Electronic Design, Cambridge, UK). Three successive electrical stimulations of the sciatic nerve at $2 \mathrm{~mA}(0.25-0.5 \mathrm{~Hz}, 0.1 \mathrm{~ms}$ duration) were initiated immediately proximal to the tibial, sural and common 
peroneal branches via a stimulus isolator (WPI Inc.) connected to the Micro 1401. After recording, the sciatic nerve was axotomized distal to the site of concentric electrode placement and the proximal end of the sciatic nerve was stimulated to ensure CMAPs were generated from direct nerve stimulation. Conduction velocity was calculated after determining the latency of CMAP onset relative to the stimulus artifact induced by electrical stimulation of the sciatic nerve and the distance between recording and stimulating electrodes measured directly on the nerve. Peak CMAP amplitude and CMAP duration were calculated from each stimulation paradigm. The average of the stimulations of the sciatic nerve for each paradigm were obtained and then averaged across animals.

Heat hypersensitivity assessments. Mice at 3-4 weeks old were acclimatized individually in a box of at least $15 \times 12 \times 10 \mathrm{~cm}^{3}$ in dimensions for $15 \mathrm{~min}$. Mice were then gently held and both hindpaws were immersed into a $50^{\circ} \mathrm{C}$ hot water bath. Latency (in s) to withdrawal from the heat stimulus was determined. Three trials were performed with a 5 -min interval in between each trial. The average of these three trials was then determined to obtain the baseline withdrawal latency. Next, mice were anesthetized under 3\% isoflurane in order to induce cutaneous inflammation. Using a syringe with a $30 \mathrm{G}$ needle, approximately $10 \mu \mathrm{L}$ of $3 \%$ carrageenan (in $0.9 \% \mathrm{NaCl}$ ) was injected into the right hairy hindpaw skin. $24 \mathrm{~h}$ later, thermal withdrawal latency using the methods described above were measured again to determine heat hypersensitivity (withdrawal latency) post inflammation ${ }^{59,60}$.

Morphometric analysis. The morphometric measurements in the early postnatal Zeb2 $\mathrm{iKO}$ experiment and the axon regeneration/remyelination assays were performed in Toluidine blue-stained semi-thin sections $(0.5 \mu \mathrm{m})$. An entire sciatic nerve cross section per animal was reconstructed by merging several high magnification photographs taken by bright field microscopy at $100 \times$ magnification. The quantifications of the 1:1 promyelinating cells, myelinated fibers and numbers of axon were performed by manually counting all of the figures present in the entire sciatic nerves cross section using the cell counter plugin from ImageJ software (National Institutes of Health).

The number of myelinated axons per nerve, the G-ratio and the number of unmyelinated axons in Remak bundles were analyzed in ultrathin sections using a JEOL 1200 EXII or Hitachi H7650 electron microscope. Quantification was done by manually counting myelinated or unmyelinated axons present in the sciatic nerves using the cell counter plugin from ImageJ software. For G-ratio analysis, images were calibrated based on the scale bar corresponding to the magnification used for each micrograph. The axons (without myelin) and fiber areas (including myelin) of randomly chosen fibers were measured, except for those surrounded by a Schmidt Lanterman incisure. The axon and fiber diameters were estimated as

$$
2 \sqrt{\frac{\text { area }}{\pi}}
$$

and the G-ratio was calculated as axon diameter/fiber diameter. At least 100 nerve fibers per animal ( $n=3$ for each group) were analyzed.
For quantification of labeled cells in transfection studies, since the ZEB2, $\mathrm{ZEB}^{2 \mathrm{R} 22 \mathrm{G}}$ and $\mathrm{ZEB}^{\Delta \mathrm{CtBP}}$ expression plasmids carry a GFP-reporter, GFP ${ }^{+}$ cells represent ZEB2 or ZEB2-mutant transfected cells. The quantification was performed by manually counting antibody co-labeled cells based on the total number of transfected cells indicated by GFP expression.

Statistical analysis. All analyses were done using Microsoft Excel or GraphPad Prism 6.00 (San Diego California, http://www.graphpad.com). Data are shown in dot plots as mean \pm s.e.m. or as box-and-whisker plots; $P<0.05$ is deemed statistically significant. Data distribution was assumed to be normal, but this was not formally tested. Count data was assumed to be non-parametric, and appropriate statistical tests were used. Statistical analysis was performed by two-tailed unpaired Student's $t$ tests, Mann-Whitney test, one-way ANOVA with post-hoc analysis by Tukey's multiple comparison test, two-way repeated measures ANOVA with Sidak's multiple comparisons test, or as indicated. Quantifications were performed from at least three experimental groups in a blinded fashion. No statistical methods were used to predetermine sample sizes, but our sample sizes are similar to those generally employed in the field. No randomization was used to collect all the data; they were quantified blindly. No animals or data points were excluded from analyses.

\section{A Supplementary Methods Checklist is available.}

Data availability. All the RNA-seq data have been deposited in the NCBI Gene Expression Omnibus (GEO) under accession number GSE74381. The data that support the findings of this study are available from the corresponding author upon reasonable request.

51. Brockes, J.P., Fields, K.L. \& Raff, M.C. Studies on cultured rat Schwann cells. I. Establishment of purified populations from cultures of peripheral nerve. Brain Res. 165, 105-118 (1979).

52. Lu, Q.R. et al. Common developmental requirement for Olig function indicates a motor neuron/oligodendrocyte connection. Cell 109, 75-86 (2002).

53. Emig, D. et al. AltAnalyze and DomainGraph: analyzing and visualizing exon expression data. Nucleic Acids Res. 38, W755-62 (2010).

54. Salomonis, N. et al. Alternative splicing regulates mouse embryonic stem cell pluripotency and differentiation. Proc. Natl. Acad. Sci. USA 107, 10514-10519 (2010).

55. Yu, Y. et al. Olig2 targets chromatin remodelers to enhancers to initiate oligodendrocyte differentiation. Cell 152, 248-261 (2013).

56. Becker, W. Recent insights into the function of DYRK1A. FEBS J. 278, 222 (2011).

57. Trapnell, C., Pachter, L. \& Salzberg, S.L. TopHat: discovering splice junctions with RNA-Seq. Bioinformatics 25, 1105-1111 (2009).

58. Trapnell, C. et al. Transcript assembly and quantification by RNA-Seq reveals unannotated transcripts and isoform switching during cell differentiation. Nat. Biotechnol. 28, 511-515 (2010).

59. Ririe, D.G., Bremner, L.R. \& Fitzgerald, M. Comparison of the immediate effects of surgical incision on dorsal horn neuronal receptive field size and responses during postnatal development. Anesthesiology 109, 698-706 (2008).

60. Marsh, D., Dickenson, A., Hatch, D. \& Fitzgerald, M. Epidural opioid analgesia in infant rats I: mechanical and heat responses. Pain 82, 23-32 (1999). 


\section{Corrigendum: Zeb2 recruits HDAC-NuRD to inhibit Notch and controls Schwann cell differentiation and remyelination}

Lai Man Natalie Wu, Jincheng Wang, Andrea Conidi, Chuntao Zhao, Haibo Wang, Zachary Ford, Liguo Zhang, Christiane Zweier, Brian G Ayee, Patrice Maurel, An Zwijsen, Jonah R Chan, Michael P Jankowski, Danny Huylebroeck \& Q Richard Lu

Nat. Neurosci.; doi:10.1038/nn.4322; corrected online 20 June 2016

In the version of this article initially published online, Danny Huylebroeck was listed under affiliation 2 (Institute of Pharmacology and Toxicology, College of Pharmaceutical Sciences, Zhejiang University, Hangzhou, China) instead of affiliation 3 (Department of Cell Biology, Erasmus University Medical Center, Rotterdam, the Netherlands). The error has been corrected for the print, PDF and HTML versions of this article. 